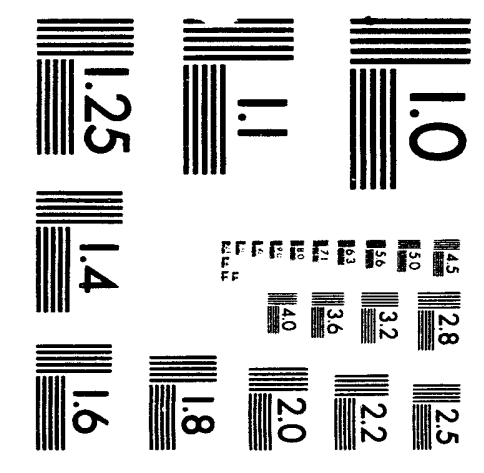



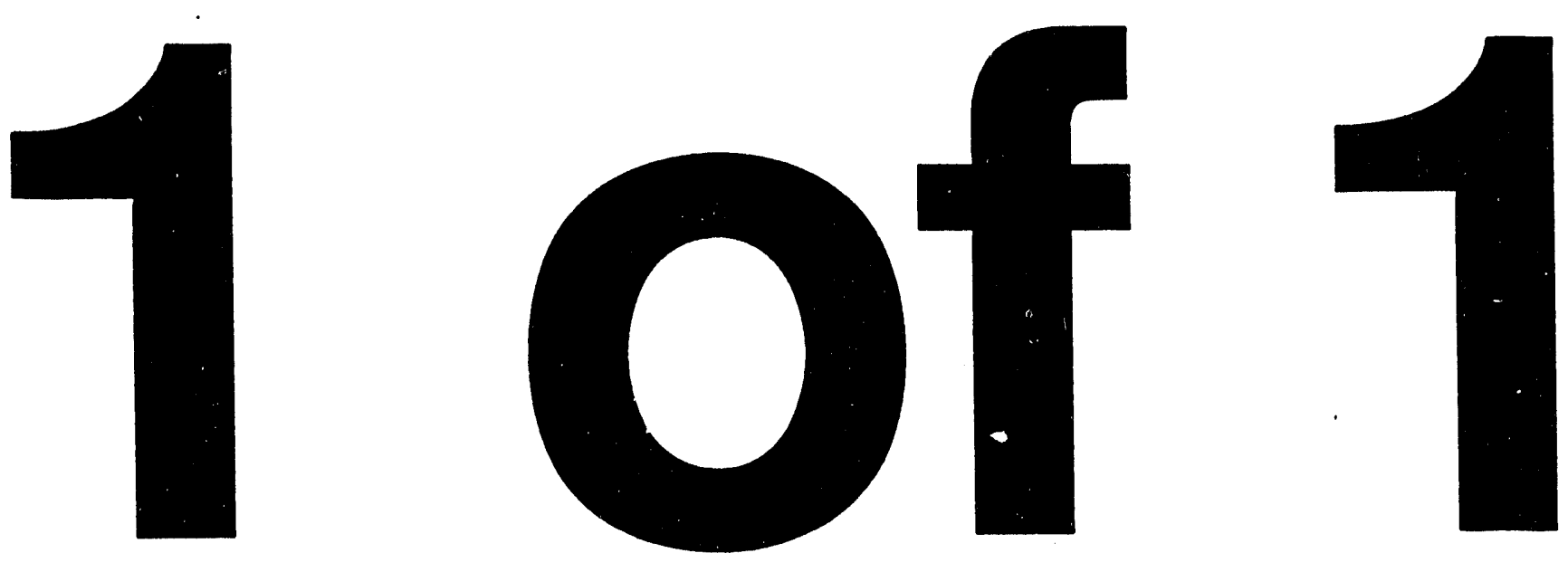


\section{NOTICE}

This report was prepared as an account of work sponsored by an agency of the United States Government. Neither the United States nor any agency therenf. nor any of their employees, makes any warranty, expressed or implied, or assumes any legal liability or responsibility for any third party's use or the results of such use of any information, apparatus, product or process disclosed in this report, or represents that its use by such third party would not infringe privately owned rights.

Printed in the United States of America

Available from

National Technical Information Service

U.S. Department of Commerce

5285 Port Royal Road

Springfield, VA 22161

NTIS Price codes

Printed copy: A03

Microfiche copy: A01 


\title{
Photon counting spectroscopy as done with a Thomson scattering diagnostic
}

\author{
D. J. Den Hartog and D. E. Rupperta)
}

University of Wisconsin-Madison, Department of Physics, Madison, Wisconsin 53706 USA

a) Permanent address: Dartmouth College, Department of Physics \& Astronomy, Hanover, New Hampshire 03755 USA

(Received

\section{ABSTRACT}

The measurement and reduction of photon counting spectral data is demonstrated within the context of a Thomson scattering diagnostic. This diagnostic contains a microchannel plate (MCP) photomultiplier tube (PMT) as the photon sensing device. The MCP PMT is not an ideal photon sensor; the loss of photoelectrons at the MCP input and the broad charge pulse distribution at the output add to the uncertainty in recorded data. Computer simulations are used to demonstrate an approach to quantification of this added uncertainty and to develop an understanding of its source; the methodology may be applicable to the development of an understanding of photon detectors other than an MCP PMT. Emphasis is placed on the Poisson statistical character of the data, because the assumption that a Gaussian probability distribution is a reasonable statistical description of photon counting data is

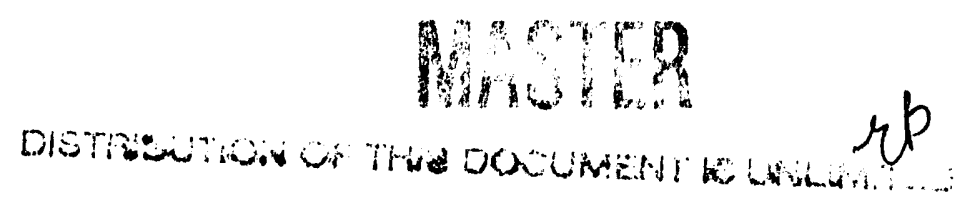


often questionable. When the count rate is low, the product of the possible number of photon counts and the probability of measurement of a single photon is usually not sufficiently large to justify Gaussian statistics. Rather, because probabilities of measurement are so low, the Poisson probability distribution best quantifies the inherent statistical fluctuations in such counting measurements. The method of maximum likelihood is applied to derive the Poisson statistics equivalent of $\chi^{2}$. A Poisson statistics based data fitting code is implemented using the Newton-Raphson method of multi-dimensional root finding; we also demonstrate an algorithm to estimate the uncertainties in derived quantities.

PACS numbers: $07.65 .-b, 06.20 . D k, 42.80 . Q y, 52.70 . \mathrm{Kz}$ 


\section{INTRODUCTION}

The measurement and reduction of photon counting spectral data presents several substantial technical and theoretical challenges. The first of these is the procurement of a detector sensitive to single photons. Choices range from the traditional photomultiplier tube to microchannel plate devices to the recently developed vacuum avalanche photodiode. The decision made usually balances factors such as speed, spectral response, size (of the device and the sensitive area), and cost. For our application we have chosen a microchannel plate (MCP) photomultiplier tube (PMT). In this paper we will examine how this device detects photons and amplifies the resulting tiny signal to a level that can be easily recorded. The specific physical mechanisms are unique to the MCP PMT, but our aim is to illustrate (by example) straightforward methods by which to understand and quantify the performance of detectors sensitive to single photons.

However, the photon detector itself is only really useful when the data it produces is correctly interpreted and reduced. A description of the algorithms necessary to do this is the second major part of this paper. This second part cannot stand without the first; proper and efficient photon counting data reduction is simply a further application of the Poisson statistics used to model and understand photon detectors. This unity simply reflects the fact that the inherent statistical fluctuations in counting data are correctly described by the Poisson probability distribution. More specifically, in the limit of small numbers $(\leqslant 10)$ of counts, the raw data cannot be correctly and robustly reduced by assuming that the symmetric Gaussian probability distribution is an acceptable approximation of the asymmetric Poisson probability distribution. This assumption is often made in order to simplify data analysis, ${ }^{1}$ but we will 
show that this is not necessary by demonstrating a straightforward application of the method of maximum likelihood entirely consistent with the Poisson statistical character of the raw data.

Since the results of this paper are set within the context of our Thomson scattering diagnostic ${ }^{2}$, which is used on the MST reversed-field pinch, a brief introduction is in order. This diagnostic is used to measure the temperature and density of electrons in a plasma, particularly the plasmas in fusion research devices. This diagnostic is simple in concept (see references such as Sheffield ${ }^{3}$ or Hutchinson ${ }^{4}$ ), but the difficulty in application arises because the total cross section for Thomson scattering is a small $6.65 \times 10^{-29} \mathrm{~m}^{2}$. In practice this typically means that one must reconstruct the Gaussian-shaped scattered spectrum from about 100 photon counts. The primary contributor to uncertainty in the derived quantities of temperature and density is the statistical uncertainty that is described by the Poisson statistics of photon counting.

This paper divides naturally into two major sections, the first on the hardware of photon detection and the second on proper and efficient data reduction. We use as an example our Thomson scattering diagnostic, but the principles thus illumined should be useful to others working in the general area of photon counting spectroscopy.

\section{PHOTON DETECTION AND COUNTING}

\section{A. Introduction}

The particular photon detector used in our Thomson scattering diagnostic is an ITT F4149 microchannel plate (MCP) photomultiplier tube (PMT) (see Fig. 1). This device records the scattered light spectrum at the exit plane of the spectrograph. This "Z-plate" MCP PMT can detect single 
photons and is in many respects similar to a traditional discrete dynode PMT. However, use of an MCP PMT as a photon detection device raises unexpected difficulties because it takes to an extreme some of the undesirable characteristics of a traditional PMT. Primary among these is a very broad charge pulse distribution at the output in response to single photon counts at the input. Of course, an MCP PMT has tremendous advantages in speed and compactness, but we will concentrate on understanding the difficulties and compensating for them. We have taken an empirical and heuristic approach to the problems encountered; thus at least the approach taken (if not the knowledge gained) may be of help to users of photon detectors other than the specific device examined.

\section{B. Description of an MCP PMT}

An MCP is an electron multiplier. When used with a photocathode at the input of the MCP and a collection anode at the output, the resulting device is a very compact PMT. The MCP itself is essentially a glass disc (or stack of glass discs) penetrated by a large number of holes (channels) of uniform micron-size diameter. The channel walls are coated with a semiconducting layer that supplies the secondary electrons necessary to produce a cascade of electrons from a single input electron. The potential between the input and output of the MCP causes the electrons to move toward the output. The electrons do not move in a straight line toward the output, but tend to bounce off the walls of the channel many times during the trip down the channel. This behavior is analogous to that of the electrons in the dynode chain of a traditional PMT. The key difference is that in an MCP, the distance between "dynodes" (electron encounters with the channel wall) is approximately 25 microns. ${ }^{5}$ This is much smaller 
than the dynode spacing in a traditional PMT and allows for MCP electron multiplication of $10^{6}$ in the space of a few millimeters.

In order to understand the factors that determine the photoncounting performance of an MCP PMT, we will follow the production of a pulse of electrons from a single photon. The sequence of events is as follows:

1) A single photon impinges upon the photocathode. Our MCP PMT has a multi-alkali photocathode with good red response (important because we use a ruby laser as the source of scattered photons). The quantum efficiency is approximately $6.5 \% @ 700$ nanometers. This means that, on average, 6.5 out of every 100 incident photons produce a photoelectron. 2) If a photoelectron is produced at the surface of the photocathode, it is accelerated by a potential of about 100 volts toward the entrance of the MCP. One of three events can occur when the photoelectron reaches the entrance of the MCP:

a) The photoelectron passes directly into a channel and begins to cascade down the dynode chain.

b) The photoelectron hits the material between adjacent channels and is lost.

c) The photoelectron hits the material between adjacent channels and ejects a secondary electron(s) which then passes into a channel(s) and begins a cascade(s).

According to research done at ITT5, a number of factors including tube geometry, construction materials, and accelerating potential determine the number of photoelectrons incident on the entrance to the MCP that are completely lost. In our MCP PMT, the ratio of channel area to MCP web face area is about $2 / 3$. In this paper we will make the pessimistic 
assumption that $15 \%$ of the incident photoelectrons are completely lost. 3) Once an electron has entered a channel, it still may not produce a cascade and subsequent pulse of electrons at the output of the MCP. The average first dynode gain is about 2.9. The simplest way to describe this situation is to assume the number of secondary electrons generated by each photoelectron is distributed according to a Poisson distribution with a mean of 2.9. This assumption is not strictly valid,6,7 but is a reasonable first approximation. For such a distribution, the probabilities of generating between 0 and 6 secondary electrons are shown in Fig. 2(a). Notice that the probability that no secondary electrons will be generated is substantial; approximately $5.5 \%$ of the incoming electrons will be lost at the first dynode. The situation worsens at the second and following dynodes because they have a mean gain of only about 1.35 . This gain factor is much smaller that the first dynode gain because the electrons pick up only about $40 \mathrm{eV}$ between channel wall bounces, whereas the incoming photoelectron hits the first dynode with well over $100 \mathrm{eV}$. The probabilities for generation of secondary electrons at the following dynodes are shown in Fig. 2(b). Note the nearly $26 \%$ probability that the "bounce" electron incident on the wall will be lost. The figure of interest is the total percentage of photoelectrons lost; i.e., the percentage of photoelectrons which enter the MCP yet do not produce a pulse of electrons at the output of the MCP. This problem can be solved in a number of ways, one being the derivation of a recursion relationship 6 and another being a Monte Carlo calculation. The results of the latter method will be discussed in Section C.

4) In a proximity focused device such as our ITT F4149, the charge collection anodes are placed close to the output of the MCP, the two 
being on the order of one millimeter apart. This configuration minimizes the spatial and time spread of the electron cloud that exits the MCP. This maintains the high speed and high spatial resolution of the MCP PMT and insures that little or no information is lost as the pulse of electrons travels to the collection anodes.

5) The collection anodes should be held near ground in order to maintain the performance of the MCP PMT. We couple the anodes of our F4149 directly into a resistive $50 \mathrm{ohm}$ load on the input to a fast video amplifier. This drives the coaxial cable leading to the $50 \mathrm{ohm}$ input of the digitizer.

6) Our diagnostic does not make use of the traditional photon counter with its adjustable bias discriminator level. Instead, we use a LeCroy $2250 \mathrm{~L}$ current integrating digitizer. This digitizer integrates the current from the anodes of the MCP PMT during a time window around the event of interest, namely, the ruby laser pulse. The number of dark counts that occurs during the 100 ns time window is very nearly zero. In all likelihood, then, all current pulses reaching the digitizer during the time window were indeed caused by photons. But, for an MCP, the size distribution of the output charge pulses for single input photoelectrons has a very broad maximum and a low peak-to-valley ratio. See Fig. 3 for the actual pulse height distribution measured for our tube. The FWHM is $\sim 130 \%$ and the peak-to-valley ratio is $1.5: 1$.

The broad distribution of output charge pulses adds uncertainty to the number of counts recorded by the digitizer (a figure that is directly proportional to the number of photons incident on the MCP PMT). There is a certain amount of irreducible uncertainty in the number of counts because this is a photon counting operation. However, the broad charge 
pulse distribution adds uncertainty to the figure that might be derived from the obvious application of Poisson statistics. The impact of a broad charge pulse distribution on the uncertainty in the number of photons recorded will be elucidated in the following two sections.

\section{Simulation of an MCP PMT}

A fairly sophisticated computer model of the performance of the microchannel plate was developed by Guest. 8 This model managed to reproduce many of the experimentally measured characteristics of a single MCP operating in non-saturated charge pulse conditions. However, the results of Guest are of limited use to us because our MCP is built in a Z-plate configuration (three single microchannel plates stacked together) which is operated in a saturated charge pulse condition. This condition complicates the model tremendously and made it necessary to incorporate empirical result's into our simulation.

The simulation of our MCP PMT is divided into two connected but separately achievable tasks. The first task is a determination of the fraction of incident photons that actually produce charge pulses at the anode of the MCP PMT. As was shown in the previous section, it is not a matter of simply multiplying the number of incident photons by the quantum efficiency of the photocathode. Some photoelectrons are lost at the entrance to the MCP and some are lost because the gain at each dynode stage is small. In order to determine the extent of this loss, a program was written to simulate the cascade of an electron down a channel of the MCP.

This simulation program followed a group of 10000 photoelectrons as they cascade down the MCP one by one. Poisson statistics governed the 
emission of secondary electrons at each dynode stage. In other words, at the first stage, the number of secondary electrons generated came from a Poisson distribution with a mean of 2.9. At the second and following stages, the number of secondary electrons generated for each cascade electron came from a Poisson distribution with a mean of 1.35. The Fortran, routine that performed this task is called POIS. ${ }^{9}$ The subroutine used to generate the Poisson deviates was adapted from the routine POIDEV listed in Numerical Recipes. 10

The key to the use of the routine POIS is to balance CPU time requirements with the need to let the electrons cascade down enough dynodes to allow accurate determination of the charge pulse distribution at the end of the dynode chain. Lombard and Martin 6 suggest that the shape of the charge pulse distribution is determined in the first few stages of electron multiplication. This point can be intuitively justified; after the first few stages the number of electrons in the cascade becomes large enough to wash out the detail of the individual Poisson events. In order to assure that the simulation cascaded down enough stages, the routine POIS was run several times with differing numbers of dynode stages. The most important result was that $25 \%$ of the input electrons were lost in the first few stages of electron multiplication. As discussed above, this substantial loss percentage is not unexpected, given the low gain of the MCP dynode stages. Fig. 4 is a histogram of the charge pulse distribution for the case with 35 dynodes, which is more than enough stages to accurately determine the charge pulse distribution. It does not show the peak evident in the measured charge pulse distribution for our Zplate MCP. This is because the model in the routine POIS does not include charge pulse saturation effects. When a charge pulse reaches a threshold 
space charge density it saturates the gain of the MCP. This changes the charge pulse distribution from a negative exponential to the quasiGaussian shape evident in the experimental curve (Fig. 3). For a good description of the details of this mechanism, see Wiza. ${ }^{11}$

Note that the actual charge pulse distribution (Fig. 3) appears to combine elements of operation in both saturated and non-saturated modes. The steep rise of the curve below the valley of the distribution is most likely due to charge pulses that do not saturate on their cascade down the MCP.12 This hypothesis is supported by the observation that the distribution curve below the valley retains the exponential character seen in the simulations that resulted in Fig. 4.

Since it is difficult to accurately simulate space charge saturation effects with a program like POIS, we used it only to quantify the number of input electrons lost in the first few dynode stages of the MCP. This information then became part of a second different simulation program which provides a better representation of the actual charge pulse distribution produced at the output of the MCP. This use of POIS is reasonable because the percentage of electrons lost is largely determined in the first few stages of the MCP dynode chain where space charge saturation has not yet occurred.

The second simulation program, called MCPOUT $^{9}$, starts with sets of photons incident on the photocathode of the MCP PMT. (This simulation is structured this way not only because it is computationally convenient but also because it is in fact the way in which we use the MCP PMT to detect Thomson scattered light. Each set of photons corresponds to a pulse of the laser.) The simulation program draws statistics from groups of identically sized sets. By letting the information from individual sets 
pass through the simulation of the MCP PMT and then gathering the output information for groups of sets, we can determine the uncertainty (or "noise") introduced by the MCP.

The program MCPOUT is conveniently divided into five sections. As an example of its function, we will track one of the sets with a mean size of 1000 photons through the simulation.

1) The actual number of photons in this set is a random deviate drawn from a Poisson distribution of mean 1000. A variation of POIDEV was used to determine all the Poisson deviates in this simulation program.

2) The number of photoelectrons produced at the photocathode is a random deviate drawn from a Poisson distribution with a mean of the actual number of photons in the set times the quantum efficiency $(6.5 \% @ 700$ $\mathrm{nm})$.

3) The number of these photoelectrons that actually survive to produce charge pulses at the anode of the MCP PMT is determined with the use of a uniform random distribution between zero and one. We know that, on average, about $85 \%$ of the photoelectrons survive and enter the MCP and, from the POIS results, about $75 \%$ of those entering the MCP actually produce charge pulses. A number between zero and one is drawn for each photoelectron incident on the MCP. If that number is greater than $0.85 \times 0.75=0.638$, then that photoelectron is lost.

4) The surviving photoelectrons cascade down the MCP and produce charge pulses. However, this program does not simulate the cascade process. It does the next best thing by choosing a size for the charge pulse caused by each single photoelectron from the measured distribution of charge pulses. The random deviate from the charge pulse distribution is drawn by the function IPHD which uses the rejection method of 
generating random deviates as described in Numerical Recipes. Tests of IPHD show that it will regenerate the measured charge pulse distribution.

5 ) Each charge pulse is converted to the number of counts that would be recorded by the LeCroy $2250 \mathrm{~L}$ digitizer. The digitizer converts at 0.5 $\mathrm{pC} /$ count.

After a group of sets has passed through the simulation of the MCP PMT, the mean and standard deviation of the number of counts can be calculated for that group of sets. For example, for 10000 sets of 1000 photons per set, the quantities in Table I were calculated. Note that the standard deviation in the initial number of photons in each set is indeed the square root of the mean. However the noise ratio (standard deviation/mean) in the digitizer counts is dominated by the noise introduced during the creation of the photoelectrons and their subsequent cascade downs the MCP. This is clearly illustrated in Fig. 5 which shows the noise ratio versus number of incident photons for set sizes ranging from 100 to 10000 photons.

\section{Discussion}

If an MCP was an ideal electron multiplier, it would introduce no noise into the information leaving the photocathode of the MCP PMT. The fluctuations in a group of digitizer count readings would have its origin mostly in the noise introduced by the Poisson nature of the photon-tophotoelectron conversion process at the photocathode. However, it is obvious from Fig. 5 that the noise introduced by photoelectron losses in the MCP and the nature of the MCP electron multiplication process is an important contribution to the magnitude of the noise ratio of a group of 
digitizer count reading.

From the above points we have seen that the fraction of photons incident on the MCP PMT that actually produce charge pulses at the anode is smaller than the quantum efficiency (6.5\%). In the simulation, only $4.1 \%$ of the incident photons actually produced charge pulses at the anode of the MCP PMT.

The broad distribution of charge pulses produced by the MCP adds to the difficulties encountered when reducing data taken with an MCP PMT. Operation of the MCP PMT as part of the Thomson scattering system requires the empirical determination of the calibration coefficient relating digitizer counts to a number of photoelectrons. Poisson statistics can then be applied to determine the uncertainty in the number of counts collected in a given wavelength channel. This calibration coefficient could be determined with a standard source such as a calibrated tungsten lamp. However, as discussed below, this method could lead to an incorrect determination of the uncertainty in the number of counts because it neglects the noise introduced by the electron multiplication in the MCP. A better method for calculation of this conversion factor uses the fact that repeated measurements of the intensity of a constant source will be distributed according to Poisson statistics. Following the lead of Hart et al.,13 let

$$
\begin{aligned}
& D \quad=\text { number of digitizer counts } \\
& C \quad=\text { conversion factor } \\
& P \quad=\text { number of photoelectrons } \\
& \sigma \quad=\text { standard deviation of } D
\end{aligned}
$$

which implies

$$
P=C D
$$




$$
\sigma=\frac{1}{C} \sqrt{P}
$$

This can be solved to yield

$$
C=\frac{D}{\sigma^{2}}
$$

Note that $P$ from this procedure is derived from the standard deviation of the number of digitizer counts and is therefore sensitive to the noise introduced by the charge pulse distribution (Fig. 3).

It is instructive to calculate the conversion factor $C$ from the simulation results shown in Fig. 5. For this simulation, each group of identically sized sets contained enough sets such that the total number of photons in each group is approximately $10^{7}$. A value of $C$ can be calculated for each group of sets of photons. Each calculated value of $C$ represents simulation data from approximately $10^{7}$ photons. The results are graphed in Fig. 6(a); the average value of $C$ is 2.18 . It is obvious that the conversion factor $C$ is best determined by groups containing a large number of sets of photons. A group containing a large number of sets of photons will provide a well-defined mean and standard deviation, and thus a well-defined value for $C$. The accuracy to which $C$ can be measured is strongly dependent on the number of sets of photons in a given group of measurements, and largely independent of the number of photons in each of those sets.

These points are illustrated in Figs. 6(b) and 7. Fig. 6(b) is the result of a simulation similar to that described above, except that this time $C$ was calculated for groups which each contained 10000 sets of photons. The number of photons in each set was stepped from 100 to 20 000. Notice that scatter in the calculated values of $C$ remains constant no 
matter how many photons are in each set. In fact, the uncertainty in $C$ is proportional to $1 / \sqrt{N}$, where $N$ is the number of sets of photons in each measurement group. This is illustrated in Fig. 7, the results of another simulation. The root-mean-square deviation of the calculated values of $C$ is plotted versus $1 / \sqrt{N}$ for groups varying in size from 34000 sets in each group to 100 in each group. The set size was fixed at 10000 photons. Actual measurements have not achieved the accuracy predicted by Fig. 7, but the real experimental setup contains additional sources of noise such as a non-ideal light source.

Use of the conversion factor $C$ that we have just calculated to convert a number of digitizer counts to a number of "photoelectrons" gives a surprising result. This derived number of "photoelectrons" (hereafter termed "effective photoelectrons") is much smaller than the true number of photoelectrons that actually produce charge pulses. In fact, the "effective quantum efficiency" for conversion of incident photons to effective photoelectrons is about $2.9 \%$. (This can be calculated by using Eq. 1 with $D$ from Table $I$ and $C=2.18$.) The effective quantum efficiency is 2.2 times smaller than the actual quantum efficiency at the photocathode of the MCP PMT. There is no subtle physics in this result, it is just a consequence of the fact that our definition in Eq. 1 makes $P$ sensitive to the noise introduced by the charge pulse distribution.

Obviously the conversion factor $C$ is not the correct factor to use when converting digitizer counts into a number of real photoelectrons. This leaves us with the question of how best to determine the uncertainty in the number of counts recorded in a given wavelength channel of the spectrograph. The solution is simple because by definition the effective photoelectrons obey Poisson statistics. The effective photoelectrons can 
be thought of as Poisson counts with the immediate result that the uncertainty is simply the square root of the given number of counts. For the purpose of quantifying uncertainty we don't need to know the number of real photoelectrons; what we want to measure is the number of effective photoelectrons.

\section{E. Summary}

This study has shown that the efficiency for conversion of incident photons to charge pulses on the anodes of the MCP PMT is probably closer to $4.1 \%$ than the $6.5 \%$ that would seem initially obvious from the photocathode quantum efficiency specification. In addition, it has been shown that the noise caused by the electron multiplication process in the MCP PMT increases the uncertainty beyond what would be calculated in a simple Poisson analysis. Also, unless care is taken and the difference between what we have termed "real" and "effective" photoelectrons is understood, significant underestimates of the actual photon count and actual count uncertainty are likely when an MCP PMT is used in conjunction with a digitizer that integrates the current pulses from the output of the MCP PMT.

\section{A POISSON STATISTICS BASED APPROACH TO DATA}

\section{REDUCTION}

\section{A. Introduction}

In the rest of this paper we will demonstrate the analysis method we have developed to reduce raw data in which the uncertainty is dominated by counting statistics fluctuations described by the Poisson probability distribution. The argument against approximating the Poisson distribution with the Gaussian distribution in this situation will be made 
explicit with the use of parameters from our Thomson scattering diagnostic as an example. The problem of "fitting" raw data to an assumed parent distribution will be solved with an application of the method of maximum likelihood that is compatible with the Poisson character of the raw data. We will also demonstrate a method to quantify the uncertainties in derived quantities.

Since the example of analysis of Thomson scattering data will be used to illustrate general principles relating to the reduction of Poisson counting data, we review some of the physics behind Thomson scattering data. In application as a diagnostic, the process of Thomson scattering (abbreviated as "TS") refers to the scattering of laser-produced monochromatic photons by free electrons in a plasma. When a photon is Thomson-scattered by a free electron in the plasma, the wavelength of the photon is Doppler-shifted by an amount proportional to the velocity of the electron. A Maxwellian distribution of electron velocities (usually a valid assumption in fusion research devices) yields a Gaussian distribution of scattered photon wavelengths. The density and temperature of the electrons are then, respectively, proportional to the area and width squared of the Gaussian distribution.

For the specific example of our Thomson scattering diagnostic, a pulsed ruby laser is fired into the plasma and the scattered photons are counted in five channels, each collecting over a particular wavelength band. The goal of the data analysis procedure is to find the parent Gaussian distribution that is most likely to have given rise to the measured set of counts. Since photon counts $c_{i}$ have been collected at shifts in wavelength $\Delta \lambda_{i}$, the parent distribution is evaluated at $\Delta \lambda_{i}$. Let $u_{i}$ be the values of the parent distribution at $\Delta \lambda_{i}$. 


$$
u_{i}=A \exp \left(\frac{-\Delta \lambda_{i}^{2}}{2 b^{2}}\right)
$$

The general method of data analysis is to compare the set of $c_{i}$ with sets of $u_{i}$ until values of $A$ and $b$ are found that give the best candidate for the parent distribution.

\section{B. The Gaussian data analysis routine: TS_GAUSSIAN}

Initially, the routine we used for TS data analysis was TS_GAUSSIAN. Fundamental to the analysis algorithm in TS_GAUSSIAN is the assumption that the fluctuations in the raw data (counts in a given channel) can be described by a Gaussian probability distribution. For example, if a measurement $u$ would most accurately reflect the state of a system, the probability to record an observation $c$ is the value of the appropriate probability distribution with a mean of $u$ and a variable value of $c$. For a Gaussian probability distribution this implies

$$
d P_{G}=\frac{d c}{\sigma \sqrt{2 \pi}} \exp \left[\frac{(c-u)^{2}}{-2 \sigma^{2}}\right]
$$

where $d P_{G}$ is the probability to record an observation $c, d c$ is an interval around $c$, and $\sigma$ is the standard deviation. The result of this assumption in TS_GAUSSIAN is an algorithm which seeks values of $A$ and $b$ (Eq. 4) that minimize this equation: 


$$
\chi^{2}=\sum_{i=1}^{5}\left(\frac{u_{i}-c_{i}}{\sigma_{c_{i}}}\right)^{2} .
$$

The resulting values of $A$ and $b$ describe the most likely parent distribution if the fluctuations in the raw data can be described by a Gaussian probability distribution. Figs. $8(\mathrm{a})$ and $8(\mathrm{~b})$ are plots of the $\chi^{2}$ surface as a function of $A$ and $b$ for a typical set of TS data. Note that this surface is a shallow basin, the bottom of which the TS_GAUSSIAN algorithm must find.

\section{Poisson statistics}

Bevington 14 states that Gaussian statistics applies when

the number of possible different observations $n$ becomes infinitely large and the probability $p$ of success for each is finitely large so that $n p>>1$.

Hence, at best, it is questionable whether the assumption of a Gaussian probability distribution is applicable to TS and other photon counting data. For example, each pulse of our TS laser contains approximately $10^{19}$ photons. Only about $10^{1}$ or less are collected in each wavelength channel of the TS diagnostic with the result that $n p=10^{1}$ which is arguably not $>>$ 1.

Instead, the probability distribution that corresponds to $n p \ll<$ is much more likely to be satisfactory. This is the Poisson distribution, which Bevington 15 describes as appropriate

for the special case when the average number of successes is very much smaller than the possible number. 
Note that here we are considering the fact that the measurement fluctuations we record are not due to measurement error or an inexact counting technique, but are statistical fluctuations inherent in the sampling of random process such as photon scattering. The Poisson distribution quantifies the probabilities of such fluctuations. The data analysis routine we have developed and will describe below is in accord with the Poisson character of raw TS data and does not make the faulty assumption that the statistical fluctuations can be described with a Gaussian probability distribution.

A Poisson probability distribution of mean $u$ and variable $c$ has the functional form:

$$
P=\frac{e^{-u} u^{c}}{c !}
$$

The asymmetrical character of the Poisson distribution is apparent when the mean is small; see Bevington for graphical examples. ${ }^{16}$

\section{The Poisson statistics compatible routine: TS_POISSON}

\section{Introduction}

The first step in the development of TS_POISSON is to apply the method of maximum likelihood to the reduction of raw data best described by a Poisson rather than a Gaussian probability distribution. The method of maximum likelihood, when applied to data characterized by a Gaussian distribution, yields the quantity $\chi^{2}$ as the fit parameter. We developed the Poisson equivalent of $\chi^{2}$.

According to Bevington, the method of maximum likelihood consists 
of making the assumption that a set of data counts is more likely to come from the actual parent distribution than from any other. 17 Application of this method to our situation turned out to be straightforward. Since the probability of measuring a given value of $c_{i}$ is given by the Poisson distribution, we see that the higher the value of the Poisson distribution for a given count $c_{i}$ and value of the parent distribution $u_{i}$, the more likely the correspondence between the two. For any particular channel, the Poisson distribution takes the value:

$$
P_{i}=\frac{e^{-u_{i}} u_{i}^{c_{i}}}{c_{i} !} .
$$

However, our Thomson scattering diagnostic has five channels, so the total Poisson probability is:

$$
\boldsymbol{P}=\prod_{i=1}^{5}\left(\frac{e^{-u_{i}} u_{i}^{c_{i}}}{c_{i} !}\right) .
$$

When this quantity has been maximized by choosing the appropriate values for $A$ and $b$, we know that we have found the most likely parent distribution.

There are many ways to find the $A$ and $b$ that maximize the Poisson probability. Our choices are implemented in TS_POISSON ${ }^{9}$, which is built out of three major and two minor subroutines. The major subroutines function 
in the following order, where arrows signify the act of calling:

$$
\begin{aligned}
& \text { NEWT_RAPH } \Rightarrow \text { USRFUN } \\
& \text { SOLVSIG } \Rightarrow \text { NEWT_RAPH } \Rightarrow \text { USRFUN }
\end{aligned}
$$

NEWT_RAPH and USRFUN find the $A$ and $b$ that correspond to the best fit. SOLVSIG finds the uncertainty of the physical parameters temperature and density. NEWT_RAPH relies heavily on the minor subroutines POISSPROB and GAMMLN for the value of the Poisson probability, evaluated each iteration, in order to check up on the latest guesses for $A$ and $b$. The next two sections contain further explanations of each subroutine.

Before we begin, however, we should note some not-so-minor details. In order to develop an algorithm to analyze the raw TS data in a statistically correct manner, it was of primary importance to preserve the Poisson nature of the raw data counts as closely as possible. This is not trivial since substantial data count modification was performed in TS_GAUSSIAN. First, the raw digitizer counts were converted to effective photoelectron (Poisson) counts with the previously determined conversion coefficient $C$. Then, background plasma light and pedestal counts were subtracted from raw counts to make signal counts. Finally, the signal counts were multiplied by terms that take into account channel width and relative sensitivity. Though the first two modifications to raw counts are necessary to yield the Poisson signal counts, the final set of modifications destroys the absolute Poisson information. Only relative signal magnitudes remained.

In order to avoid this compromise of the data, data modifications in TS_POISSON are limited to tabulation of the Poisson signal counts. 
Modifying factors are lumped into a quantity, $q_{i}$, one for each channel. Instead of modifying the Poisson cuunts, these quantities appear in the inverse position on the parent population, modifying it. Hence, whenever the parent population is calculated in TS_POISSON, it is calculated with the values of $q_{i}$, viz.:

$$
u_{i}=A q_{i} \exp \left(\frac{-\Delta \lambda_{i}^{2}}{2 b^{2}}\right)
$$

Though the $q_{i}$ have not been mentioned until now in order to avoid confusion, this consideration is very important; it is one of the underpinnings of TS_POISSON.

A straightforward but necessary detail is the set of formulas to find the physical quantities temperature and density (density is directly proportional to the area under the Gaussian). The formula for the temperature, $T_{e}(e V)=0.530 b^{2}$, may be derived from Sheffield 18 with $\theta=$ $90^{\circ}$ and $\lambda=694.3 \mathrm{~nm}$. The formula for area in TS_POISSON is $A b \sqrt{2 \pi}$. (The calibration coefficient for conversion of area to density can be determined with a Raman scattering technique, ${ }^{19}$ but this has been done only occasionally with our Thomson scattering diagnostic. Results will be presented in this paper in terms of area.)

Now, on to an explanation of the subroutines.

\section{NEWT_RAPH}

Following Bevington, we derived a set of equations that had as their 
solution the $A$ and $b$ that would maximize the Poisson probability of Eq. 9. These equations were derived by the simple process of setting the partial derivatives of the Poisson probability equal to zero. Differentiation with respect to $A$ and $b$ yields 9

$$
\begin{aligned}
& \sum_{i=1}^{5}\left(u_{i}-c_{i}\right)=0 \\
& \sum_{i=1}^{5}\left(u_{i}-c_{i}\right) \Delta \lambda_{i}^{2}=0 .
\end{aligned}
$$

The roots of this set of equations maximize the Poisson probability.

To find these roots, we chose the Newton-Raphson method. 20 Starting with initial guesses for the values of $A$ and $b$, the NewtonRaphson subroutine manipulates two matrices to produce the $\delta A$ and $\delta b$ by which it will modify its guesses. After each update of $A$ and $b$ the process starts over again, until $\delta A$ and $\delta b$ pass beneath some preset limit.

Because we have two equations and two unknowns, we have only $2 x$ 1 and $2 \times 2$ matrices. The first is the matrix of values that Eqs. 11 and 12 take on for the $A$ and $b$ in question. The second is the matrix of derivatives of the two equations, each with respect to $A$ and $b$. The values of the components of these matrices are calculated in USRFUN. The matrix manipulation required takes place in NEWT_RAPH in the four lines following the call to USRFUN. For a derivation of the elements of the matrices, as well as a derivation of the proper matrix manipulation, see Ref. 9.

Note that the matrix manipulation encoded in NEWT_RAPH is a substitute for that which is otherwise done by two separate Numerical 
Recipes subroutines. 20 If more parameters were included one would probably want to refer to these routines for the necessary $L U$ decomposition.

The minor subroutine POISSPROB calculates the Poisson probability for a single channel. It is always called in a loop, once for each channel, so that the entire probability may be evaluated. Note that the individual constituents of the Poisson distribution can become very large. As a precaution, parameters used in calculations are made double precision. Another precaution against overflow is the splitting up of the individual terms of the distribution. For example $u^{c}$ appears as $\left(10^{c}\right)(u / 10)^{c}$. GAMMLN is used by POISSPROB to calculate $c$ !. See POISSPROB ${ }^{9}$ and Numerical Recipes for the details.

We have found that the Newton-Raphson method does an exemplary job of finding the roots of Eqs. 11 and 12. An example of the results obtained is shown in Figs. 9(a) and 9(b). These are plots of the surface of the Poisson probability (Eq. 9) with respect to $A$ and $b$ for the same set of TS data used to illustrate $\chi^{2}$ in Figs. $8(a)$ and $8(b)$. Note that the Poisson probability maximum is sharply peaked and well-defined in comparison to the shallow minimum exhibited by $\chi^{2}$.

\section{SOLVSIG}

This routine calculates the uncertainty in the values of plasma electron temperature and density. For a certain quantity $x$, the uncertainty in $x$ is 


$$
\sigma_{x}=\left[\sum_{i=1}^{5} \sigma_{c_{i}}^{2}\left(\frac{\delta x}{\delta c_{i}}\right)^{2}\right]^{\frac{1}{2}}
$$

where, in our case, $c_{i}$ are Poisson counts and $\sigma_{c_{i}}$ are the uncertainties in $c_{j}$. Strictly speaking, TS_POISSON should use the square roots of $c_{i}$ for $\sigma_{c_{i}}$ in the calculation of the derived uncertainties. But this is not correct because the $\sigma_{c_{l}}$ are increased by the fact that the $c_{i}$ come from the subtraction of the background plasma light signal from a total (raw) light signal. Therefore, we estimate the uncertainties $\sigma_{c_{1}}$ as the square root of the total light signal plus the background plasma light signal.

The derivative in Eq. 13 can be approximated numerically by changing the counts in a channel by a particular amount and finding the new value of $x$. We employ the "five point method" for taking numerical derivatives. This method is accurate, but a bit cumbersome, viz.:

$$
\frac{\delta x}{\delta c_{i}}=\frac{x\left(c_{i}-\Delta\right)-8 x\left(c_{i}-2 \Delta\right)+8 x\left(c_{i}+2 \Delta\right)-x\left(c_{i}+\Delta\right)}{12 \Delta} .
$$

NEWT_RAPH is called in SOLVSIG to find the new $A$ and $b$ corresponding to the change made in counts.

\section{E. Results: TS_POISSON versus TS_GAUSSIAN}

An examination of the derived values for temperature and area calculated by TS_GAUSSIAN and TS_POISSON reveals that differences exist. Table $\|$ is a compilation of the temperatures and areas for twenty 
nominally identical shots. Note that the two algorithms calculate mean values of temperature, area, and their associated uncertainties that are nearly equal. However, the values of these derived quantities for any one shot can differ substantially.

By definition, the best fits to the raw TS data are achieved by maximizing the Poisson probability. In a sense it is the fundamental parameter by which "goodness-of-fit" should be judged, no matter what fitting algorithm is being used to reduce Thomson scattering data. TS_POISSON always produces the fit that maximizes the Poisson probability, TS_GAUSSIAN does not. The difference in the Poisson probability at the "best fits" determined by each algorithm for a given shot can be substantial. As an example, see Fig. 10 for contour plots of the Poisson probability and the $\chi^{2}$ surface for a typical shot. Contrast this to shot illustrated in Figs. 8(a) and 9(a) in which the best fits are almost exactly the same. This difference arises from the fact that the fitting routine in TS_POISSON is much more robust than that in TS_GAUSSIAN.

Consider the raw spectra shown in Figs. $11(\mathrm{a})$ and $11(\mathrm{~b})$. These spectra are from the nominally identical shots used to illustrate Figs. 9 and 10. Plotted in these figures are the raw data and the best fit provided by TS_POISSON. In Fig. 11(a), the raw data counts are aligned almost perfectly to the shape of the parent Gaussian distribution; any fitting routine should have an easy time. The counts in Fig. 11(b), however, cannot so easily be imagined as reflecting a Gaussian parent distribution. This is most likely due simply to statistical (Poisson) fluctuations in the raw data and not to a different parent distribution. In this and similar cases, the fit provided by TS_POISSON is simply more believable than that derived by TS_GAUSSIAN. The fitting algorithm in TS_POISSON is based upon 
the assumption that the raw data will exhibit Poisson statistical fluctuations; the fitting algorithm in TS_GAUSSIAN is not. In short, for shots in which the raw data is a good reflection of the parent distribution, the difference between TS_GAUSSIAN and TS_POISSON should be, and is, very small. However, on shots where statistical fluctuations cause a poor representation of the parent distribution, it is only the algorithm based on Poisson statistics that produces a believable fit to the raw data.

\section{F. Summary}

For analysis of low count spectra such as produced by a Thomson scattering diagnostic, a fitting algorithm based on maximization of the Poisson probability is demonstrably superior to the usual $\chi^{2}$ algorithm based on the Gaussian probability distribution. The methods described in this paper should have application not only to Thomson scattering, but to the analysis of any count rate dominated spectra.

\section{G. Future work}

The parent Gaussian distribution to which the raw data is fit (Eq. 10 ) is valid only for Thomson scattering from a low temperature plasma $\left(T_{e} \leq 500 \mathrm{eV}\right)$. At higher temperatures, relativistic effects enter. The first order effect is a shift of the center of the Thomson scattered spectrum. 21 Equation 10 and the fitting routine TS_POISSON should be modified if temperatures higher than $500 \mathrm{eV}$ are to be measured.

\section{CONCLUSION}

Simple computer simulations can be used to quantify the non-ideal characteristics of an MCP PMT. Two major contributors of uncertainty (or 
noise) to recorded data are the loss of photoelectrons at the MCP input and the broad charge pulse distribution produced at the output in response to a photoelectron at the input. Since the measurement fluctuations in photon counting spectral data are fundamentally Poisson in character, the correct way to reduce it and fit it to the appropriate parent distribution is to maximize the Poisson probability of the fit.

\section{ACKNOWLEDGEMENTS}

The authors gratefully acknowledge helpful conversations with $M$. Cekic, E. A. Den Hartog, S. A. Hokin, D. J. Holly, and S. C. Prager and thank them for a careful reading of this manuscript.

This work was supported by the U. S. Department of Energy.

1Philip R. Bevington, Data Reduction and Error Analysis for the Physical Sciences (McGraw-Hill, New York, 1969), p. 111.

2D. J. Den Hartog, Ph.D. thesis, University of Wisconsin-Madison, 1989.

3 John Sheffield, Plasma Scattering of Electromagnetic Radiation (Academic, New York, 1975), p. 46.

4. H. Hutchinson, Principles of plasma diagnostics (Cambridge University, Cambridge, 1987), p. 239.

${ }^{5} \mathrm{E}$. H. Eberhardt, "Parameters pertaining to microchannel plates and microchannel plate devices," ITT Electro-optical Products Division Technical Note No. 127 (ITT, Fort Wayne, 1980).

${ }^{6}$ Francis J. Lombard and Fred Martin, Rev. Sci. Instrum. 32, 200 (1961). 
7 Andrew T. Young, in Methods of Experimental Physics (Academic Press, New York, 1974), Vol. 12, Part A, Chap. 1.

${ }^{8}$ A. J. Guest, Acta Electronic 14, 79 (1971).

${ }^{9}$ See AIP document no. PAPS for 14 pages of MCP simulation code, data reduction code, derivation, and matrix manipulation. Order by PAPS number and journal reference from American Institute of Physics, Physics Auxiliary Publication Service,335 East 45th Street, New York, NY 10017. The price is $\$$ _ for each microfiche (98 pages) or $\$$ _ for photocopies up to 30 pages, and $\$ \_$for each additional page over 30 pages. Airmail additional. Make checks payable to the American Institute of Physics.

10 William H. Press, Brian P. Flannery, Saul A. Teukolsky, and William T. Vetterling, Numerical Recipes: The Art of Scientific Computing (Cambridge University, Cambridge, 1986), p. 207.

11 Joseph Ladislas Wiza, Nucl. Instrum. Methods. 162, 587 (1979).

12Private communication from Richard J. Hertel, ITT Corporation, Electro-optical Products Division, Fort Wayne, Indiana, U.S.A.

13G. W. Hart, F. M. Levinton, and D. H. McNeill, "A study of the effects of photon statistics on Thomson scattering data," Princeton Plasma Physics Laboratory Report No. PPPL-2293 (Princeton Plasma Physics Laboratory, Princeton, 1985).

14Bevington, p. 43.

${ }^{15}$ Bevington, p. 36.

16 Bevington, p. 41. 
17 Bevington, p. 101.

${ }^{18}$ Sheffield, p. 45.

${ }^{19}$ S. C. McCool, I. L. McCool, R. D. Bengtson, and P. E. Phillips, "Calibration of Thomson scattering density measurements," Fusion Research Center Report No. 234 (University of Texas at Austin, Austin, 1981).

20press, p. 269.

21 Sheffield, p. 200. 


\begin{tabular}{|l|c|c|c|}
\hline \multicolumn{1}{|c|}{ Quantity } & Mean & $\begin{array}{c}\text { Standard } \\
\text { deviation }\end{array}$ & Noise ratio \\
\hline \hline Photons & 999.94 & 31.6 & 0.0316 \\
\hline $\begin{array}{l}\text { Photoelectrons at } \\
\text { photocathode }\end{array}$ & 65.0 & 8.27 & 0.127 \\
\hline $\begin{array}{l}\text { Photoelectrons producing } \\
\text { charge pulses }\end{array}$ & 41.4 & 6.54 & 0.158 \\
\hline Digitizer counts & 13.2 & 1.46 & 0.186 \\
\hline
\end{tabular}

Table I. The results of passing 10000 sets of 1000 photons per set through the simulation MCPOUT. The "noise ratio" is the standard deviation divided by the mean. 
Temperature (eV)

Shot \# TS_GAUSSIAN TS_POISSON

\begin{tabular}{ccccccccc} 
Shot \# & \multicolumn{2}{c}{ TS_GAUSSIAN } & TS_POISSON & \multicolumn{2}{c}{ TS_GAUSSIAN } & \multicolumn{2}{c}{ TS_POISSON } \\
\hline 6 & 353 & \pm 81 & 332 & \pm 72 & 340 & 36 & 359 & \pm 36 \\
\hline 8 & 433 & 84 & 428 & 81 & 476 & 39 & 485 & 39 \\
\hline 9 & 384 & 76 & 368 & 70 & 494 & 43 & 508 & 43 \\
\hline 12 & 318 & 52 & 320 & 52 & 580 & 43 & 589 & 43 \\
\hline 14 & 319 & 66 & 326 & 66 & 524 & 45 & 543 & 46 \\
\hline 17 & 290 & 44 & 295 & 43 & 633 & 46 & 664 & 47 \\
\hline 18 & 289 & 50 & 281 & 48 & 456 & 40 & 465 & 40 \\
\hline 20 & 335 & 76 & 390 & 93 & 495 & 44 & 517 & 46 \\
\hline 24 & 317 & 51 & 312 & 50 & 561 & 43 & 570 & 44 \\
\hline 27 & 334 & 56 & 338 & 57 & 631 & 46 & 635 & 46 \\
\hline 29 & 284 & 37 & 285 & 37 & 752 & 50 & 753 & 50 \\
\hline 31 & 311 & 51 & 303 & 49 & 523 & 42 & 532 & 43 \\
\hline 33 & 307 & 50 & 303 & 48 & 489 & 40 & 494 & 40 \\
\hline 37 & 463 & 142 & 460 & 139 & 339 & 38 & 344 & 38 \\
\hline 38 & 385 & 119 & 334 & 92 & 307 & 37 & 336 & 37 \\
\hline 41 & 360 & 86 & 355 & 83 & 371 & 36 & 389 & 37 \\
\hline 44 & 433 & 98 & 442 & 100 & 489 & 42 & 493 & 42 \\
\hline 46 & 208 & 37 & 207 & 37 & 307 & 31 & 307 & 31 \\
\hline 47 & 402 & 101 & 416 & 106 & 278 & 31 & 283 & 32 \\
\hline 54 & 263 & 55 & 279 & 61 & 393 & 38 & 399 & 38 \\
\hline Mean & 339 & 71 & 339 & 69 & 472 & 41 & 483 & 41 \\
\hline$\sigma_{\text {mean }}$ & 63 & & 63 & & 126 & & 125 &
\end{tabular}

Table II. A comparison of temperature and area as calculated by TS_GAUSSIAN and TS_POISSON for a series of low density shots. The row labeled Mean is the average of the quantity in the column above; $\sigma_{\text {mean }}$ is the standard deviation of that quantity. Note that the $\sigma_{\text {mean }}$ for area is much larger than the mean uncertainty in area; this is indicative of substantial plasma density fluctuations during sawtooth events. 


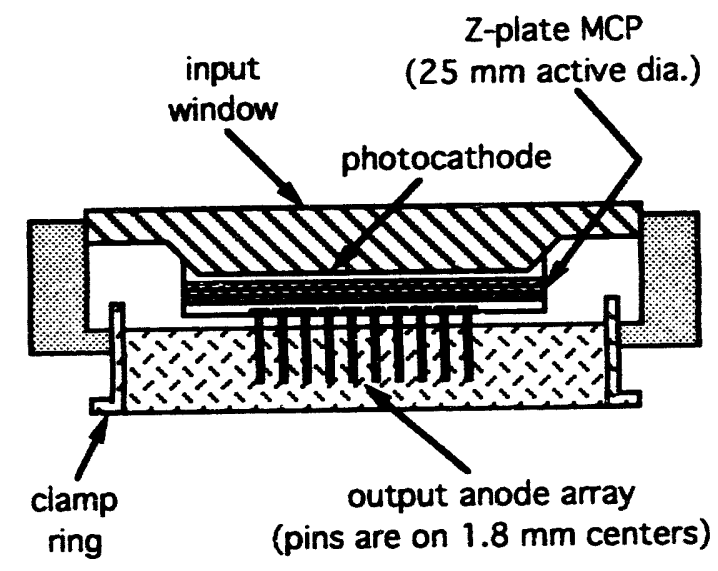

FIG. 1. An illustration (not to scale) of the ITT F4149, a multi-anode microchannel plate photomultiplier tube.
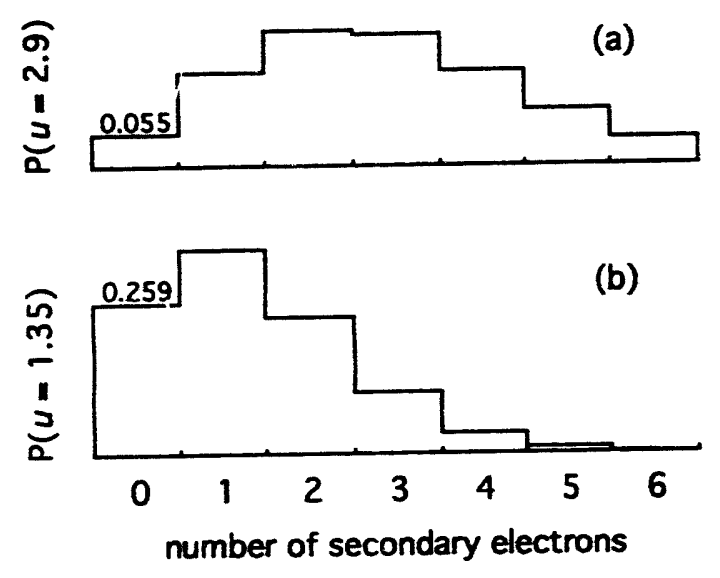

FIG. 2. The probabilities for generation of secondary electrons at (a) the first dynode and (b) following dynodes. The mean of the Poisson distribution is $u$, the probability for zero secondary electrons is shown to indicate the scale of the histograms. 


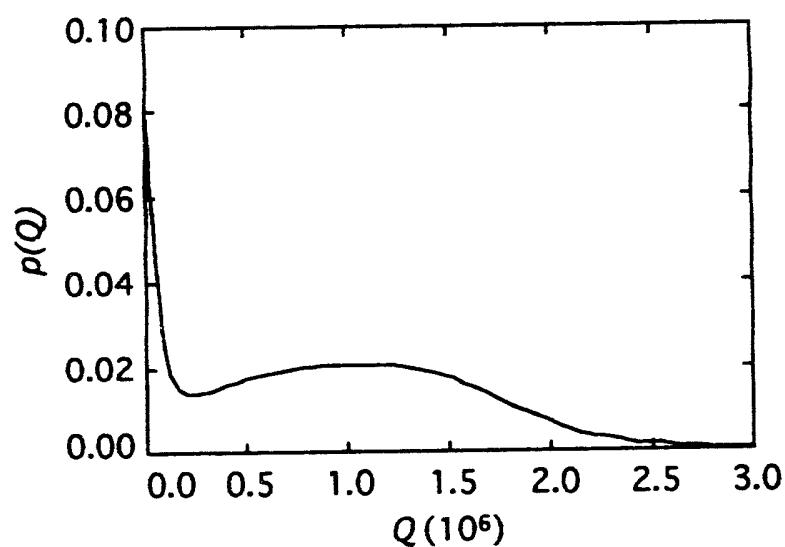

FIG. 3. The measured charge pulse distribution for our MCP PMT. The MCP was biased at $2400 \mathrm{~V}$. $Q$ is the number of electrons in an output charge pulse. Each output charge pulse is presumably caused by one input electron. The probability of measuring a charge pulse of size $Q$ is given by $p(Q) \Delta Q$ where $\Delta Q$ is a small interval around $Q$.

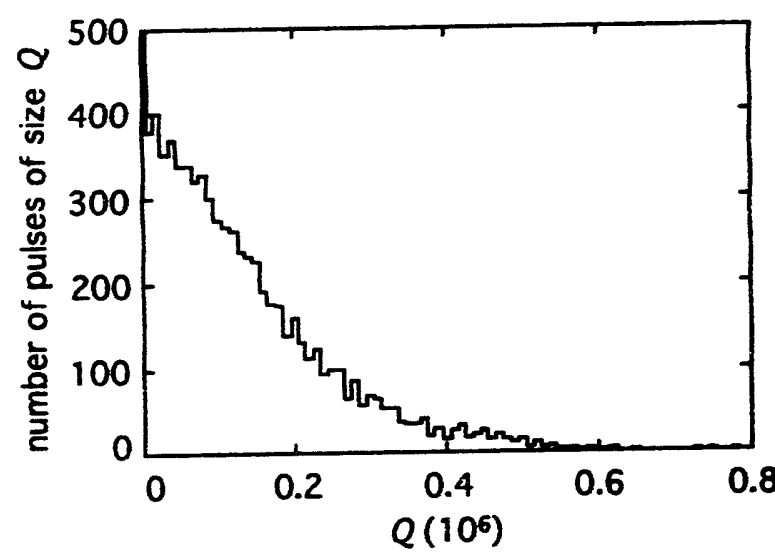

FIG. 4. The charge pulse distribution from the simulation with 35 dynodes. $Q$ is the number of electrons in an output charge pulse. The large number of pulses with $Q=0$ is not shown on the graph. 


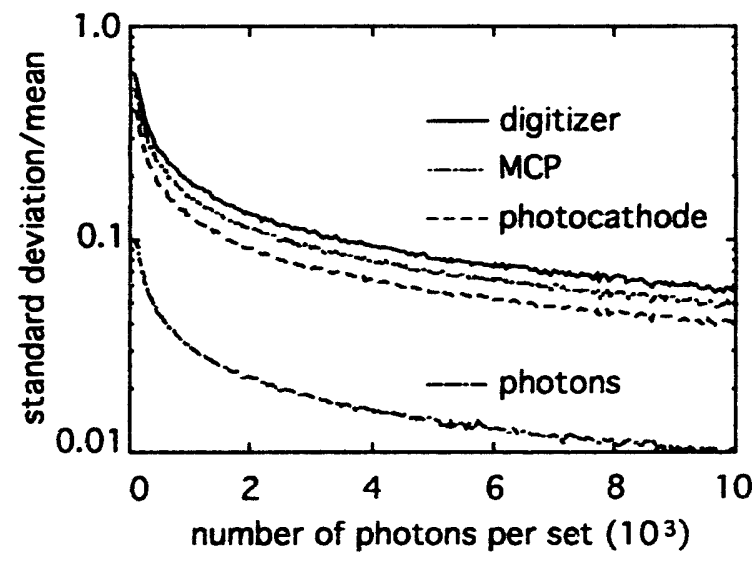

FIG. 5. The noise ratio (standard deviation/mean) at various stages of the electron multiplication process versus number of incident photons.
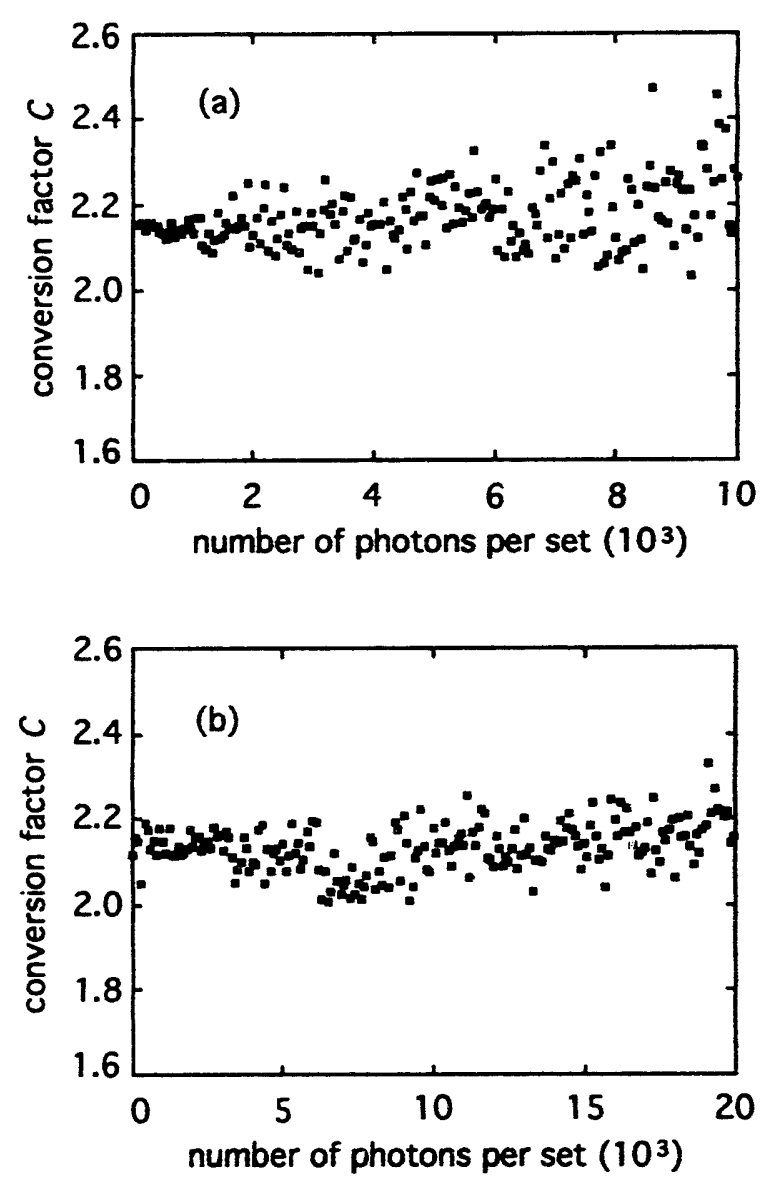

FIG. 6. The conversion factor $C$ for each group of sets; each group is represented by a datapoint. In (a), the number of sets of photons in each group is equal to $10^{7}$ divided by the number of photons per set. In (b), there were 10000 sets of photons in each group. 

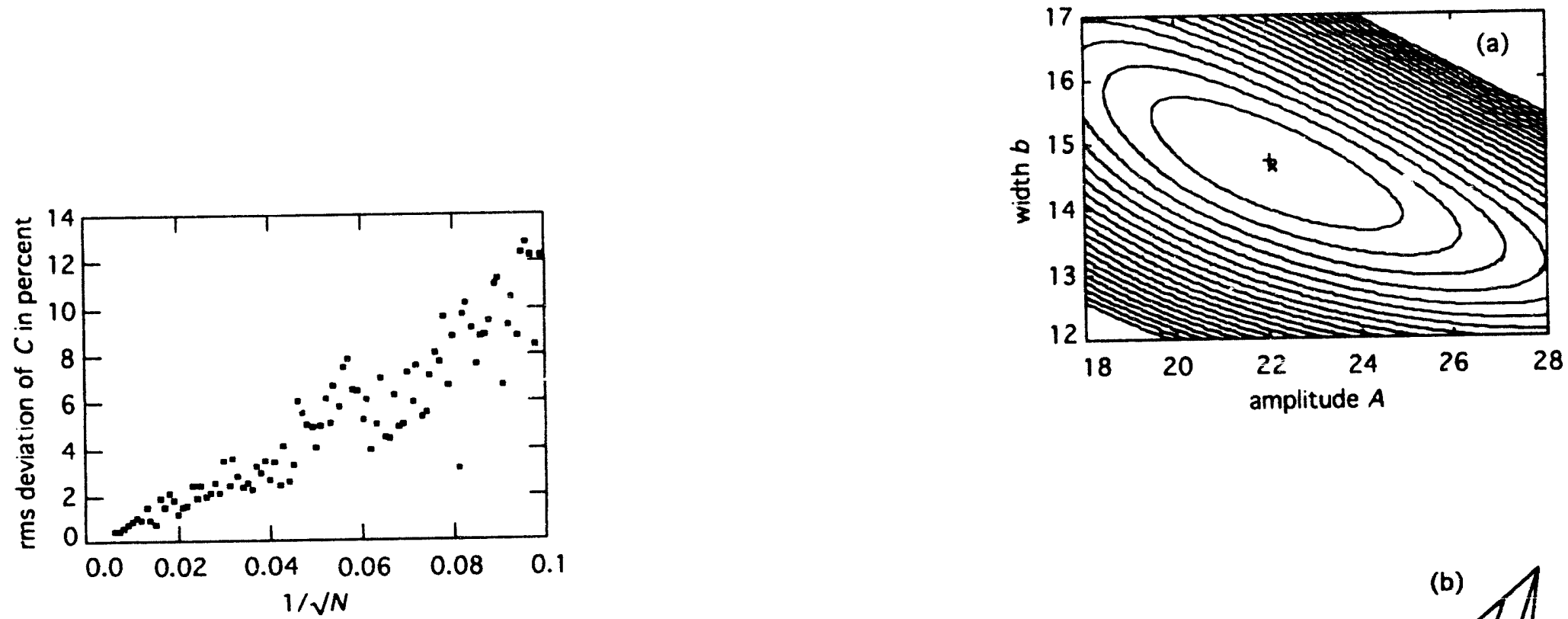

FIG. 7. The rnis deviation of the calculated values of $C$ for each group of sets versus $1 / \sqrt{ } N$, where $N$ is the number of sets of photons in each group. $N$ ranged from 34000 sets to 100 sers. The set size was fixed at 10000 photons.

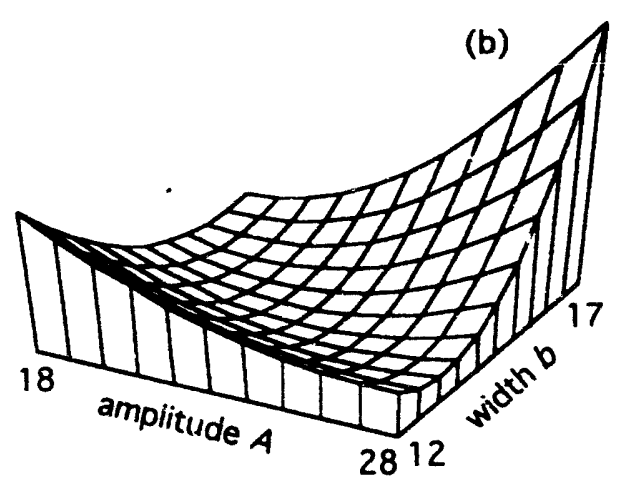

FIG. 8. A contour plot (a) and a surface plot (b) of the $\chi^{2}$ surface for a typical shot. The best fit from TS_Poisson is represented by a plus (+), the best fit from TS_GAUSSLAN by a cross $(x)$. 

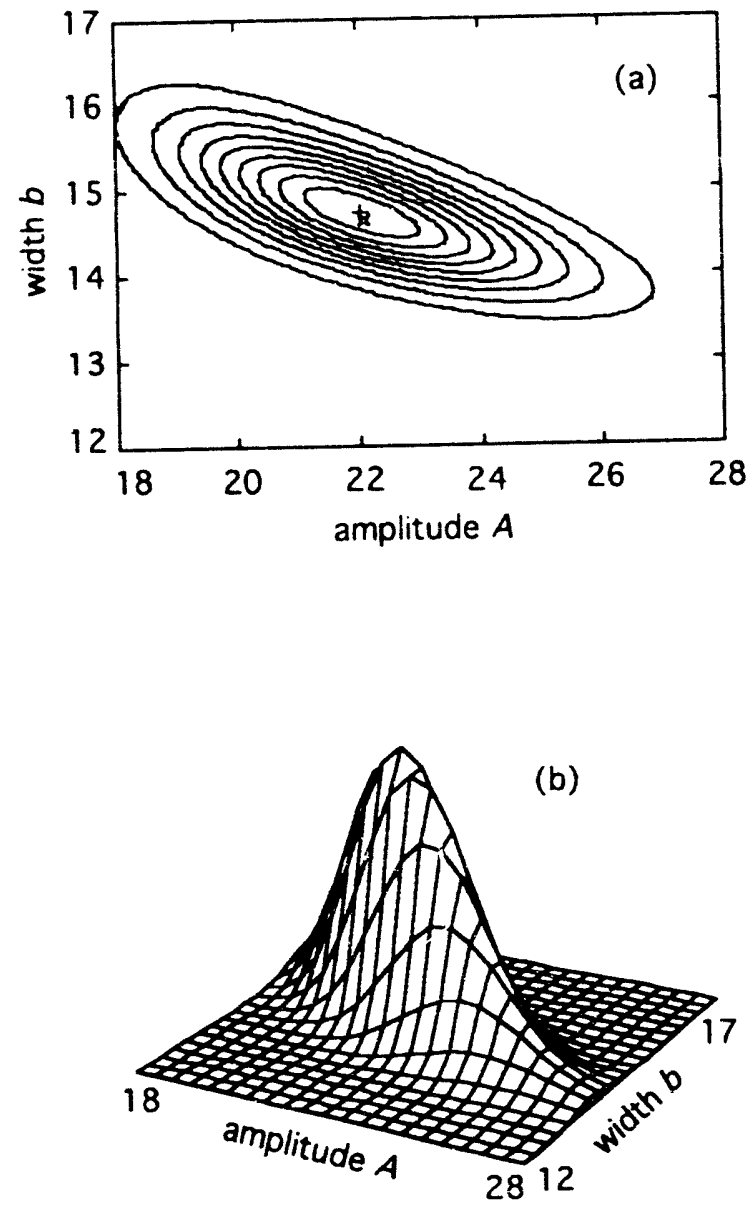

FIG. 9. A contour plot (a) and a surface plot (b) of the Poisson probability surface for the same shot as shown in Fig. 8. The best fit from TS_POAsSON is represented by a plus $(+)$, the best fit from TS_GAUSSIAN by a cross $(x)$.

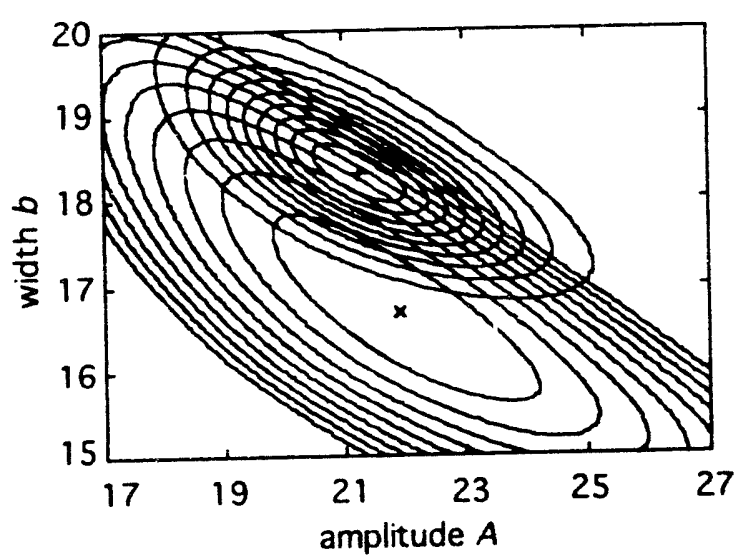

FIG. 10. A contour plot of both the Poisson probability distribution (upper contours) and the $\chi^{2}$ surface (lower contours) for a typical shot. The best fit from TS_poisson is represented by a plus $(+)$, the best fit from TS_GAUSSIAN by a cross $(x)$. 

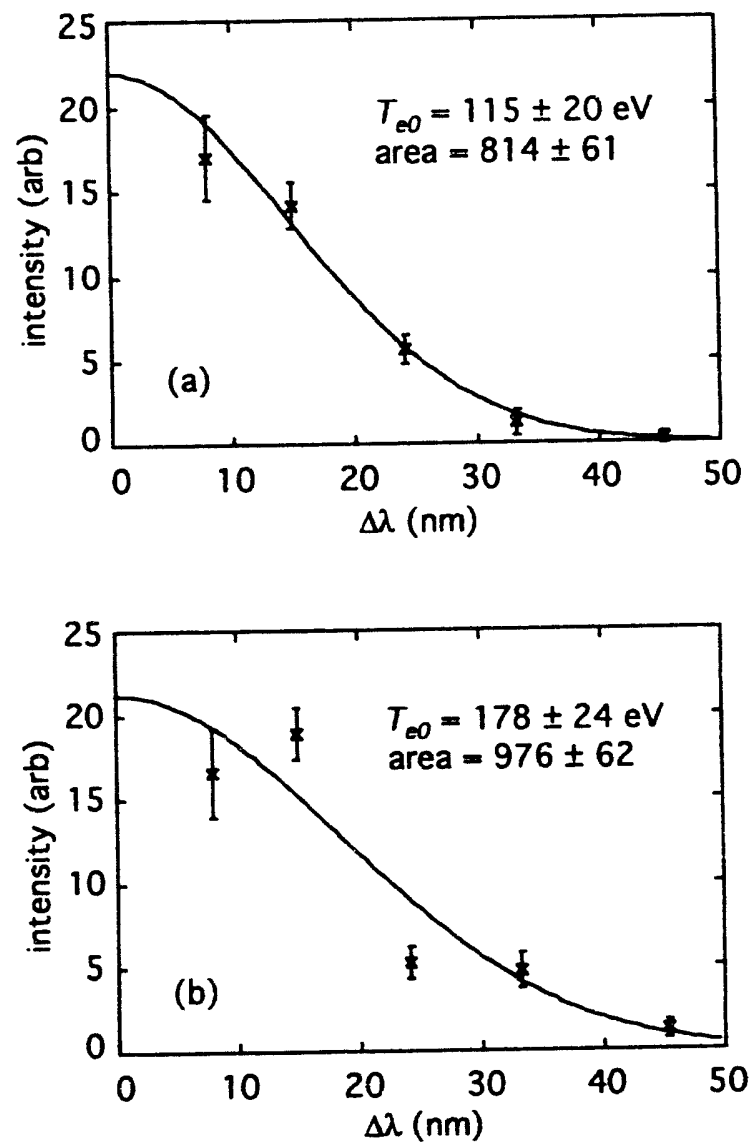

FIG. 11. The Thomson scattered spectra for two typical shots. The raw data are a (a) good

representation and (b) poor representation of the parent distributions. The Gaussian fit shown was derived by TS_POISSON. 
I. Derivation of equations used in NEWT_RAPH

Start with

$$
P=\prod_{i=1}^{5} \frac{e^{-v_{1}} u_{i}^{c_{1}}}{c_{i} !}
$$

Put into a workable form using

$$
\begin{aligned}
\ln (\mathbf{P}) & =\sum_{i=1}^{5} \ln \left(e^{--u}\right)+\sum_{i=1}^{5} \ln \left(u_{i}^{q}\right)+\sum_{i=1}^{5} \ln \left(\frac{1}{c_{i} !}\right) \\
& =\sum_{i=1}^{5}-u_{i}+\sum_{i=1}^{5} c_{i} \ln \left(u_{i}\right)+\text { constant }
\end{aligned}
$$

Take partials $\quad\left(u_{i}=A \exp \left(\frac{\gamma_{i}}{b^{2}}\right), \gamma_{i} \equiv \frac{-\Delta \lambda_{i}^{2}}{2}\right)$

$$
\begin{aligned}
\frac{\partial \ln (P)}{\partial A} & =\sum_{i=1}^{5} \exp \left(\frac{\gamma_{i}}{b^{2}}\right)+\sum_{i=1}^{5} \frac{c_{i}}{u_{i}} \exp \left(\frac{\gamma_{i}}{b^{2}}\right)+0=0 \\
& =\sum_{i=1}^{5}-\frac{u_{i}}{A}+\sum_{i=1}^{5}\left(\frac{c_{i}}{A}\right)=0 \Rightarrow \sum_{i=1}^{5}\left(c_{i}-u_{i}\right)=0 \\
\frac{\partial \ln (P)}{\partial b} & =\sum_{i=1}^{5}-u_{m}\left(\frac{-2}{b^{3}}\right)+\sum_{i=1}^{5}\left(\frac{c_{i}}{u_{i}}\right) u_{i \gamma_{i}}\left(\frac{-2}{b^{3}}\right)+0=0 \\
& =\sum_{i=1}^{5} u_{i} \frac{\Delta \gamma_{i}^{2}}{b^{3}}+\sum_{i=1}^{5} a_{i} \frac{\Delta \gamma_{i}^{2}}{b^{3}}=0 \Rightarrow \sum_{j=1}^{5}\left(c_{i}-u_{i}\right) \Delta \lambda_{i}^{2}=0 .
\end{aligned}
$$

II. Derivation of matrix elements

Adopting notation used in Numerical Recipes, choose:

$$
\begin{aligned}
& \beta_{1}=\sum_{i=1}^{5}\left(c_{i}-u_{i}\right) \\
& \beta_{2}=\sum_{i=1}^{5}\left(c_{i}-u_{i}\right) \Delta \lambda_{i}^{2}
\end{aligned}
$$

$$
x_{1}=A, \quad x_{2}=b, \quad\left(u_{i}=A \exp \left(\frac{y_{1}}{b^{2}}\right), \quad n=\frac{-\Delta \alpha^{2}}{2}\right)
$$

Hence, $\alpha_{11}=\frac{\partial \sum_{i=1}^{5}\left(-c_{i}+u_{i}\right)}{\partial A}=\sum_{i=1}^{5}\left(0+\exp \left(\frac{\gamma_{1}}{b^{2}}\right)\right) \Rightarrow \alpha_{11}=\sum_{i=1}^{5} \frac{u_{i}}{A}$

$$
\begin{aligned}
& \alpha_{12}=\frac{\partial \sum_{i=1}^{5}\left(-c_{i}+u_{i}\right)}{\partial A}=\sum_{i=1}^{5}\left(0+u_{i \gamma_{i}}\left(\frac{-2}{b^{3}}\right)\right) \Rightarrow \alpha_{12}=\sum_{i=1}^{5} u_{i} \frac{\Delta \lambda_{i}^{2}}{b^{3}} \\
& \alpha_{21}=\frac{\partial \sum_{i=1}^{5}\left[\left(-c_{i}+u_{i}\right) \Delta \lambda_{1}^{2}\right]}{\partial A}=\sum_{i=1}^{5}\left[\left(0+\exp -\left(\frac{\gamma_{i}}{b^{2}}\right)\right)\right] \Delta \lambda_{i}^{2} \Rightarrow \alpha_{21}=\sum_{i=1}^{5} \frac{u_{j} \Delta \lambda_{i}^{2}}{A} \\
& \alpha_{22}=\frac{\partial \sum_{i=1}^{5}\left[\left(-c_{i}+u_{i}\right) \Delta \lambda_{1}^{2}\right]}{\partial A}=\sum_{i=1}^{5}\left[\left(0+u_{m}\left(\frac{-2}{b^{3}}\right)\right)\right] \Delta \lambda_{i}^{2} \Rightarrow \alpha_{22}=\frac{u_{1} \Delta \lambda_{i}^{3}}{b^{3}}
\end{aligned}
$$


III. Derivation of matrix manipulation

\section{We seek to solve}

$$
\sum_{i j=1}^{2} \alpha_{i j} \delta_{j}=\beta_{i}
$$

$\alpha_{i j}$ and $\beta_{i}$ are defined above, but

$$
\begin{aligned}
& \delta_{1}=\delta_{\mathrm{A}} \\
& \delta_{2}=\delta_{\mathrm{A}} .
\end{aligned}
$$

Since we have two equations and two unknowns, the general formula above may be written as:

$$
\begin{aligned}
& \beta_{1}=\alpha_{12} \delta x_{1}+\alpha_{12} \delta x_{2} \\
& \beta_{2}=\alpha_{21} \delta x_{1}+\alpha_{22} \delta x_{2}
\end{aligned}
$$

From the second, solve for $\delta x_{2}$ :

$$
\delta x_{2}=\frac{\beta_{2}-\alpha_{21} \delta x_{1}}{\alpha_{22}}
$$

From the first, solve for $\delta x_{1}$ :

$$
\delta x_{1}=\frac{\beta_{1}-\alpha_{12} \delta x_{2}}{\alpha_{11}}
$$

Substitute $\delta \mathbf{x}_{1}$ :

$$
\begin{gathered}
\delta x_{2}=\frac{\beta_{2}-\alpha_{21}\left[\frac{\beta_{1}-\alpha_{12} \delta x_{2}}{\alpha_{11}}\right]}{\alpha_{22}} \\
\Rightarrow \delta x_{2}\left(1-\frac{\alpha_{12} \alpha_{21}}{\alpha_{11} \alpha_{22}}\right)=\frac{\left(\beta_{2}-\frac{\alpha_{21} \beta_{1}}{\alpha_{11}}\right)}{\alpha_{22}} \\
\Rightarrow \delta x_{2}=\frac{\left(\beta_{2}-\frac{\alpha_{21} \beta_{1}}{\alpha_{11}}\right)}{\alpha_{22}} \frac{\alpha_{11} \alpha_{22}}{\left(\alpha_{22} \alpha_{11}-\alpha_{12} \alpha_{21}\right)} \\
\delta x_{2}=\frac{\beta_{2} \alpha_{11}-\beta_{1} \alpha_{21}}{\left(\alpha_{22} \alpha_{11}-\alpha_{12} \alpha_{21}\right.} .
\end{gathered}
$$

Substitute back:

$$
\begin{aligned}
\delta x_{1} & =\frac{\beta_{1}-\alpha_{12}\left(\frac{\left(\beta_{2} \alpha_{11}-\beta_{1} \alpha_{21}\right)}{\left(\alpha_{22} \alpha_{11}-\alpha_{12} \alpha_{21}\right)}\right)}{\alpha_{11}}=\frac{\beta_{1-}-\alpha_{12} \delta x_{2}}{\alpha_{11}} \\
= & \frac{\left(\beta_{1}-\alpha_{22} \alpha_{11}-\beta_{1} \alpha_{12} \alpha_{21}-\beta_{2} \alpha_{11} \alpha_{12}+\beta_{1} \alpha_{12} \alpha_{21}\right)}{\alpha_{11}\left(\alpha_{22} \alpha_{11}-\alpha_{12} \alpha_{21}\right)}
\end{aligned}
$$

or

$$
\delta x_{1}=\frac{\beta_{1} \alpha_{22}-\beta_{2} \alpha_{12}}{\left\langle\alpha_{22} \alpha_{11}-\alpha_{12} \alpha_{21}\right)}
$$




\section{MCP aimulation program}

PROGRAM POIS

c simulation to determine the fraction of incident photoelectrons that $c$ actually produce charge pulses at the anode of an ITT F4149 MCP PMT.

INTEGER SAMPLE, DYNODE, NUMSTAGE

PARAMETER (SAMPLE $=10000$ )

PARAMETER (NUMSTAGE $=26$ )

INTEGER INITELEC, STAGE, INTERELEC, ISEED

INTEGER CASCADE (SAMPLE), ELEC (NUMSTAGE)

REAL INITGAIN, GAIN

$C$ opening the data files

OFEN (UNIT = 3, FILE = 'CASCADE_25. DAT' , STATUS = ' UNKNOWN'

ISEED $=$ INT $($ SECNDS $(0.0) * 100)$

INITGAIN $=2.9$

DO $I=1$, SAMPLE

The first stage has a gain of 2.9

INITELEC = IPOIDEV (INITGAIN, ISEED

C The following stages have a gain of 1.35 .

ELEC(1) = INITELEC

INTERELEC $=0$

DO $J=1, \operatorname{ELEC}(S T)$

INTERELEC = INTERELEC + IPOIDEV (GAIN, ISEED)

END DO

ELEC (STAGE+1) = INTERELEC

END DO

$C$ Writing the data to the data file

WRITE $(3, *)$ CASCADE

C Closing the data files

CLOSE (3)

END
PROGRAM MCPOUT

Co $C$ of an ITT F4149 MCP PMT.

INTEGER PH_BEG, PH_END

PARAMETER (PHLBEG $=100$ )

( PHLEND $=10000$

INTBGER*4 SAMPLE, ISAM
PARAMETER (SAMPLE $=100000$

INTEGER* TAPA 8 TOTPH, TOTPE, TOTCASC, TOTSURV

REAL'8 TOTPH, TOTPE,

INEAI AVPH, AVCASC, AVSURV. SUMPH, SUMCASC, SUMSURV, SIGPH, SIGCASC, SIGSURV

REAL AVPE, SUMPE, SIGPE

REAL QE, WE, UNIF, RSAM, COUNTS (127)

C opening the data files

OPEN (UNIT $=1$, FILE $=$ ' PHD_INT . DAT', STATUS $=$ ' OLD' ')

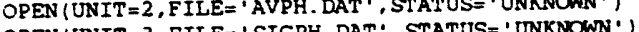

OPEN (UNT = '

OPEN (INTT = 5 , FILE =' SIGCASC. DAT', STATUS = ' UNKNOWN' ')

OPEN (UNTT =6, FILE= ' AVSURV. DAT', STATUS=' UNKNOWN')

OPEN (UNIT $=7$, FILE $=$ ' SIGSURV. DAT' ' STATOS $=$ ' UNKNOWN' ')

OPEN ( ONIT $=8$, FILE $=$ ' AVPE. DAT', STATUS= ' ONKNOWN')

OPEN (UNIT $=9$, FILE = ' SIGPE. DAT' , STATUS= ' UNKNOWN' ')

C Reading in the measured pulse height distribution for the MCP PMr. READ $(1, *)$ COUNTS CLOSE (1)

$C$ The seed for the random number generation routines. ISEED $=\operatorname{INT}(\operatorname{SECNDS}(0.0) * 100$

$\begin{aligned} \text { C 36.38 of the photoelectrons are lost. } & \text { WE }=0.363\end{aligned}$

DO PHOTON=PH_BEG, PHLEND, 50

PH = REAL (PHOTON)

C Insuring that the total number of photons in each group is $10 E 7$. ISAM = NINT (SAMPLE $/(\mathrm{PH} * 0.01)$ )

IF (ISAM . GT. SAMPLE) ISAM = SAMPLE

TOTPH $=0$

TOTPE $=0$

TOTCASC $=0$

DO $X=1$, ISAM

$C$ Determination of the actual number of photons in this set. $\operatorname{NOMPH}(K)=$ IPOIDEV $(\mathrm{PH}$, ISEED) TOTPH $=$ TOTPH + REAL (NOMPH $(R)$

Determination of the number of photoelectrons produced at the photocathode

QE $=0.065 * \mathrm{NTMPH}(\mathrm{K})$ 
$\operatorname{NUMPE}(K)=\operatorname{IPOIDEV}(Q E$, ISEED $)$

TOTPE = TOTPE + REAL (NUMPE (K))

C Determination of the number of photoelectrons that actually survive to

NUMGURV $(K)=0$
$D O J=1, \operatorname{NUMPE}(K)$$$
\text { IF }
$$

IF (UNIF. GT. WE) NUMSURV (K) $=\operatorname{NUMSURV}(K)+1$

END DO
TOTSURV = TOTSURV + REAL (NUMSURV $(K))$

C Determination of the size of the charge pulses produced by the surviving

$C$ photoelectrons.

$$
\begin{aligned}
& \text { CASCADE }(K)=0 \\
& D O J=1, \text { NUMSURV }(K)
\end{aligned}
$$

CASCADE $(R)$ = CASCADE $(K)+$ IPHD (COUNTS, ISEED)

END DO

TOTCASC = TOTCASC + REAL $(C A S C A D E(K))$$$
\text { END DO }
$$

C Calculation of the averages of the above quantities for this group of sets. RSAM $=$ REAL (ISAM)
AVPH $=$ TOTPH/RSAM

AVPE $=$ TOTPE/RSAM

AVCASC $=$ TOTCASC $/$ RSAM

AVSURV $=$ TOTSURV $/$ RSAM

C Calculation of the standard deviation of the above quantities for this

C group of sets.

SUMPH $=0.0$
SUMPE $=0.0$

SUMCASC $=0.0$

SUMSURV $=0.0$

DO $I=1$, ISAM

SUMPH $=$ SUMPH $+($ REAL $($ NOMPH $(I))-$ AVPH $) * * 2$

SUMPE $=$ SOMPE + (REAL (NOMPE(I)) - AVPE) $* 2$

SUMCASC $=$ SUMCASC + (PEAL (CASCADE (I) $)-$ AVCASC $) * * 2$

SIGPH

SIGPH $=$ SQRT (SUMPH/ (RSAM - 1))
SIGPE $=$ SORT (SUMPE / RSAM - 1))

SIGCASC $=$ SQRT (SUMCASC/(RSAM - 1))
SIGSURV $=$ SQRT (SUMSURV/ (RSAM - 1))

C Writing the data to the data file

WRITE $(2, *)$ AVPH

WRITE $(4, *)$ AVCASC

WRITE $(5, *)$ SIGCASC

WRITE $(6, *)$ AVSURV

WRITE $(7, *)$ SIGSUR

WRTE $(8, *)$ ANPE

END DO

C closing the data files

CLOSE (2)

CLOSE ( 3 )

CLOSE (4)

CLOSE $(5)$

CLOSE (6)
CLOSE (7)

CLOSE ( 8 )

CLOSE ( 9 )

END

INTEGER FUNCTION IPHD (COUNTS, ISEED)

C Returns a integer value that is a random deviate drawn from the MCP pulse $C$ height distribution. RANI (ISEED) is used as a source of uniform random

REAL COUNTS (127)

PARAMETER (AREA $=1921717.3$ )

$1 \quad X 0=-30.0 *$ LOG $((1-$ AREA $*$ RAN1 (ISEED $)) / 1.95 E 6)+1)$ IXO $=$ NINT (XO)
XO $=$ REAL (IXO)

IF (IXO.LE. O.OR. IXO.GE.127) GOTO I

YO $=6.5 E 4 * \operatorname{EXP}(-X 0 / 30). * \operatorname{RAN1}$ (ISEED)

ISUB $=$ IXO +1
IF (YO. GT.COUNTS (ISUB) ) GOTO 1

$I P H D=I X O$

RETUR 
Data reduction programs

OPTIONS/G_FLOATING

TS POISSON (input file)

Finds a best fit by maximizing the "Poisson probability." Notes explaining the purpose of each subroutine are found in each subroutine.

Thomson system parameters are read in NAMELIST form from file TSPROC. INP

- Fit definitions

I

ped = residual pedestal in the digitizer channel

tssig = the raw signel in digitizer counts

delam = delta lambda (nanometers)

light in photoelectrons

in digitizer counts

pns = plasma light noise level in photoelectrons

pnsrow = plasm light noise level in digitizer counto

tls = the light signal in photoelectrons

tlsraw the light signal in digitizer cou

Cw = the channel width in nanometers

norm = coefficient used to normalize spectrometer channels

is $=$ the Thomson scattered if the signal in photoelectrons

unc = uncertainty in $1 \mathrm{~s}$

Eh digitizer counts to photoelectrons The conversion coefficient $" C$ " is the inverse of cprop.

OUTPOT

Teo = central electron temperature in eV

$\mathrm{TeDe}=$ uncertainty in $\mathrm{TeO}$

the data; proportional to the electron density

- areaer = uncertainty in are

Dink with NEWT PAPH SOLVSIG JSBFUN POISSPPOB \&

Declarat ions:

PARAMETER YNCHEAX

REAL ped $(2 *$ NCHMAX), tissig $(2 *$ NCHMAX) (a)

A

pns (NCFMAX), pnsraw (NCHMAX)

CW (NCHMAX), Dorm (NCHMAX)

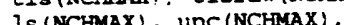

ls (Nerima

amp, amp_err, width, width_err

prob, $q$ (NCHMAX), $x(2), y x$ (NCFMYAX)

Teo, Te0_err, area, area_err

REAL*8 poissprob

- Other.

real

sqrepi/k
i, $j, k$

- Namelist input.$$
\text { /TS/ }
$$

ts_signame, nchan, cprop.
- Open and read the input file.

read (11, TS)

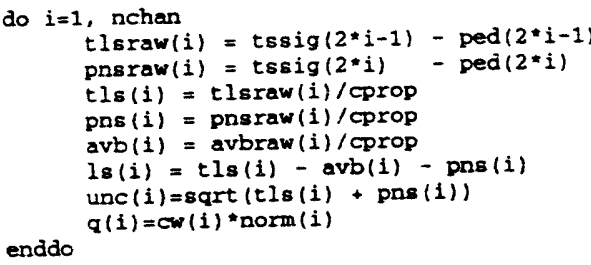

c intTIAL guesses

$x(1)=30$.
$x(2)=30$.

FIND THE Amp AND width THAT MAXIMIZE THE POISSON PROBABILITY, USING THIS SPEEDY ROOT FINDER.

amp $=x(1)$

C SOLVE FOR UNCERTAINTIES

CALL SOLVSIG $(x$, delam, $1 s, a$, unc, area_err, TeO_err)

$x(1)=\operatorname{amp}$
$x(2)=w i d t$

CALCULATE PHYSICAL QUANTITIES area $=$ sqrtpi *amp *width*squt $(2,1$

prob $=100000$

DO $i=1,5$ ENDDO

$2 * x(2) * * 2)$

type *, '

10) date, shot

, for ' 111 , SHOT ', i3)

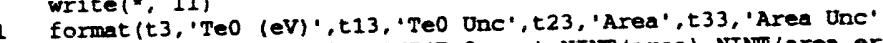

wite ( $*, 12)$ NIM

format $(t 2, i 4, t 12, i 4, t 22, i 4, t 32, i 4)$

format (t 3, 'P P prob', t23, 'Lst En'।

write (", 14) prob, NTMT (energy)

format (t2, 1 Pe10. 3, t22, i4)

END 
OPTIONS/G_FLOATING

SURROUT INE NEW_RAPH ( $x$, delam, ls, q) sum $(y x(i)-1 s(i))=0$ and sum $(y x(i)-1 s(i)) * \operatorname{eelam}(i) * * 2)=0$.

The roots $x(1)$ and $x(2)$ serve as good estimates of the $x(1)$ and $x(2)$ that will maximize the poisson probability.

simpler version of MNEWT and LUDCMP, LUBKSB (for matrices of dim. 2). chapters 9.6 and 2.3 respectively.

cailed bY TIS POISSPROB

REAL*8 old_prob, trial_prob, chngx (2), poissprob

REAL top, bottom, delam (5), $q(5)$

\& $y x$, unc $(5)$, trial, is (5), $x(2)$

\& limit,a_keep,b_keep,alpha $(2,2)$, beta(2)

INTEGER $i, j, n, n$ trial

KEEPING THE BEST PROBABILITY

a. $k e e p=x(1)$

b-keep=x(2)

DO $i=1,5$

ENDDO old_prob $=01 d$ prob * POISSPROB $(y x$, is $(i))$

nerial $=20$

Generally, Newt_Raph requires .1t. 10 D $i=1$, ntria

CALL USRFUN(alpha, beta, $x$, delam, ls, q) ! see USRFun for info on aiphas.

top=beta (1)*alpha $(2,2)-$ beta (2)*aiphs $(1,2)$ : sol'n of $2-d$ bottom=alpha $(2,2) *$ aipha $(1,1)$-alpha $(1,2) *$ alpha $(2,1)$ : system of

chngx (1) =top/bottom
chngx (2) = (beta (2)-alpha $(2,1) *$ chngx(1) /alpha $(2,2)$

if (chngx (1) .gt. 5. ) chngx (1) =chngx (1)/5. if (chngx (2).gt.5. / chngx (2)=chngx(2)/5. : too big, it would erash. $x(1)=x(1)+$ chng $x(1)$

C CALCULATE FOR NEW $x \cdot s$ THE CORRESPONDING

c PROBABILITY. IF THE NEW PROBABILITY IS GREATER THAN THE OLD THEN SAVE THE NEW VALUES OF $x$.

trial_prob $=100000$

Do $j=\overrightarrow{1}, 5$

trial $=x(1) * q(j) * \exp (-(\operatorname{delam}(j) * * 2) /(2 * x(2) * * 2))$ ENDDO

IF(trial_prob.gt.old prob)mHEN

old_prob=trial_prob

a.keep $=x(1)$

b_keep $=x(2)$

ENDIF

IF $x(1), x(2)$ ARE CHANGED BY AN AMOUNT LESS THAN 1imit, RETURN. Iimit $=.000001$

(1i) 12 limit and abs (chngx(2)). lt.iimit) THEN

$$
\begin{aligned}
& x(1)=a_{\text {_keep }} \\
& x(2)=b_{\text {_keep }}
\end{aligned}
$$

ENDIF
ENDDO

$x(1)=a_{-}$keep

$x: 2\}=$ b_keep

RETURN

END

OPT IONS / G_FLOATING

FUNCTION POISSPROB $(u, x)$

tistrion.

called by TS_POISSON, NEWT_RAPH.

Given a mean of $u$ and a particular value of $x$ GAMML calculates the natural log of the gamma function. Note that $x$ ! = garma $(x+1)$ for any $x$.

This program looks ugly because the individual components of the poisson distribution $((\exp (-u) * u * x) / x !)$ drive the computer to overflow, whereas the entire distribution, for reasonable values of $x$, is always of good listribution's components, allowing some pretty big u's and the distribucion's components, allowing some precty big u's and $x$ 's to be calculated.

David Ruppert. $5 / 27 / 93$

INTEGER Count, $i$

count $=0$

$x_{\text {_orig }}=\mathrm{x}$

u_orig $=u$

$x_{\text {_step }}=\mathrm{x}$

1 CONTINUE $! \mathbf{x}:$ is denominator of poiss dist. The computer can IF (x_step.gt.33.) THEN ! handle 33!; anything higher is tricky. Hence, x_step=x_step-1. ! shave off the components above 32, and multiply goto 1 them later.

ENDIF

Why?... How? The reason:

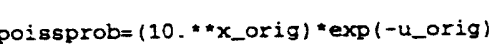

DOissprob=poissprob/exp (GAML.N (x step+1.)

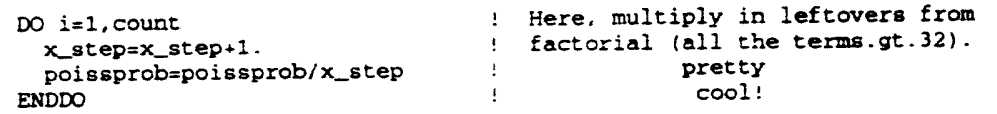

poissprob=poissprob*(u_orig/10.)**x_orig

RETURN 
SUBROUTINE SOLVSIG ( $x$, delam, $1 s, q$, unc, area_err, Te0_err)

Calculates uncertainties of area and Te 0 by the $5 \mathrm{pt}$. " numerical

C derivative method.

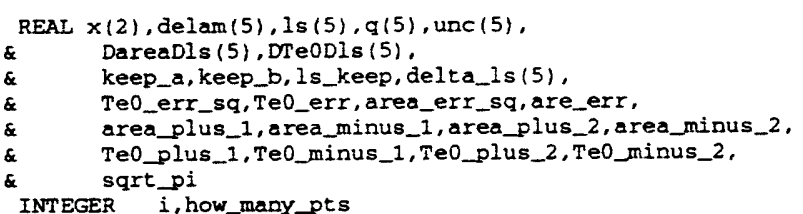

sqrt_pi=1.7724539

keep_a $a=x(1)$

keep_b=x

delta_ls(i) =sqrt (sqrt (1.d-7))*1s(i) ! from Num. Rec. ch. 5.7

if (delta_is(i) eq.0) delta_ls(i)=.05

temp $=1 s(i)+$ delta_Is $(i)$ numerical

delta_ls $(i)=$ temp-is $(i) \quad$ I derivatives.

1s_keep=ls (i)

ls $(i)=1 s_{-} k e e p+$ delta $1 s(i)$

area plus $1=\operatorname{sqrt} i^{*} \times(1)^{*} \times(2) * \operatorname{sart}(2)$

Te0 plus $1=.530 * x(2) * * 2$

$x(2)=$ keep_b

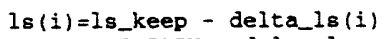

CALL NEWT_RAPH ( $x$, delam, 1s, q)

area_minus $1=$ sqrt_pi*x(1)*x(2)*sqrt $(2$.

$x(2)=$ keep_b
$x(1)=k e e p+a$

Is $(i)=1$ s_keep +2 *delta_ls $(i)$

CALI NEWT_RAPH ( $x$, delam, $1 s, q)$

area_plus_2 $=8 \mathrm{qrt} \_\mathrm{P} \times \mathrm{x}(1) \times \times(2) * \mathrm{Bqrt}(2$.)

Tec_plus_ $2=.530 \times x(2)$

$x(2)=k e e p \_$b

Is $(i)=1 s \_k e e p-2 . *$ delta_ls $(i)$

CALL

area_minus_2 $=8 q r t \_p t * x(1) * x(2) *$ sqrt $(2$.)

Te0_minus $2=.530 * x(2) * * 2$

\section{$x(2)=$ keep_b
$x(1)=$ keep_a}

$1 s(i)=1 s \_$keep

DareaDIs $(i)=$ (area_minus_2-8. *area_minus_ $1+\quad$ derivatives

8 Darea_plus_1-area_plus_2)/(12.*delta_ls(i))

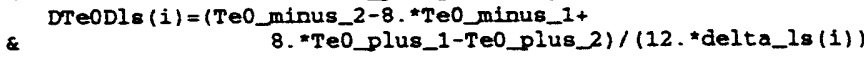

ENDDO area_err_sq $=0$.

$\mathrm{TeO}_{-}$err $-5 q=0$.

$\{=1,5$ ENDDO

area_err $=$ sqrt (area_err_sq)

RETURN

END

SUBROUTINE USRFUN (alpha, beta, $x$, delam, ls, q)

parameters $x(1)$ and $x(2)$ evaluated at $x(1)$ and $x(21)$.

calculates betas (2X1 matrix of equations evaluated at $x(1) \& x(2)$ ). These quantities yield in NEW'_RAPH the amounts by which $x(1)$ and $x(2)$ should be changed.

called by NEWT RAPH see Numerical Recipes

REAL $1 \mathrm{~s}(5)$, delam $(5), q(5), y \times(5)$,

* $\quad x(2)$, alpha $(2,2)$, beta $(2)$

DO $i=1,2$

Do $j=1.2$

beta $(i)=0$.

ENDDO $(i, j)=0$

ENDDO

$y x(i)=x(1) * q(i) * \exp (-(\operatorname{delam}(i) * * 2),(2 * x(2) * * 2)$,

ENDDO

DO $i=1,5$

beta $(1)=(1 \mathrm{~s}(i)-\mathrm{yx}(i))+$ beta $(1)$

$+\operatorname{beta}(2)$

alpha $(1,1)=x \times(1) / x(1)+$ alpha $(1,1)$

$3)+$ alpha (1,2)

ENDDO

RETURN

END 
EXTERNAL DISTRIBUTION IN ADDITION TO UC-20

S.N. Rasband, Brigham Young University

T. Dolan, EG\&G Idaho, Inc.

R.A. Moyer, General Atomics

J.B. Taylor, Institute for Fusion Studies, The University of Texas at Austin

E. Uchimoto, University of Montana

F.W. Perkins, PPPL

O. Ishihara, Texas Technical University

M.A. Abdou, University of California, Los Angeles

R.W. Conn, University of California, Los Angeles

P.E. Vandenplas, Association Euratom-Etat Belge, Belgium

Centro Brasileiro de Pesquisas Firicas, Brazil

P. Sakanaka, Institute de Fisica-Unicamp, Brazil

Mme. Monique Bex, GANIL, France

J. Radet, CEN/CADARACHE, France

University of Ioannina, Greece

S. Ortolani, Istituto Gas Ionizzati, EURATON-ENEA-CNR Association, Italy

R. Andreani, Associazione EURATOM-ENEA sulla Fusione, Italy

Plasma section, Energy Fundamentals Division Electro'echnical Laboratory, Japan

Y. Kondoh, Gunma University, Kiryu, Gunma, Japan

H. Toyama, University of Tokyo, Japan

Z. Ypsjoda. University of Tokyo, Japan

FOM-Instituut voor Plasmafysica "Rijnhuizen," The Netherlands

Z. Ning, Academia Sinica, Peoples Republic of China

P. Yang, Shandong University, Peoples Republic of China

S. Zhu, University of Science \& Technology of China, People's Republic of China I.N. Bogatu, Institute of Atomic Physics, Romania

M.J. Alport, University of Natal, Durban, South Africa

R. Storer, The Flinders University of South Australia, South Australia

B. Lehnert, Royal Institute of Technology, Sweden

Librarian, CRPP, Ecole Polytechnique Federale de Lausanne, Switzerland

B. Alper, Culham Laboratory, UK

A. Newton, UK

2 for Chicago Operations Office

5 for individuals in Washington Offices

INTERNAL DISTRIBUTION IN ADDITION TO UC-20

80 for local group and file 

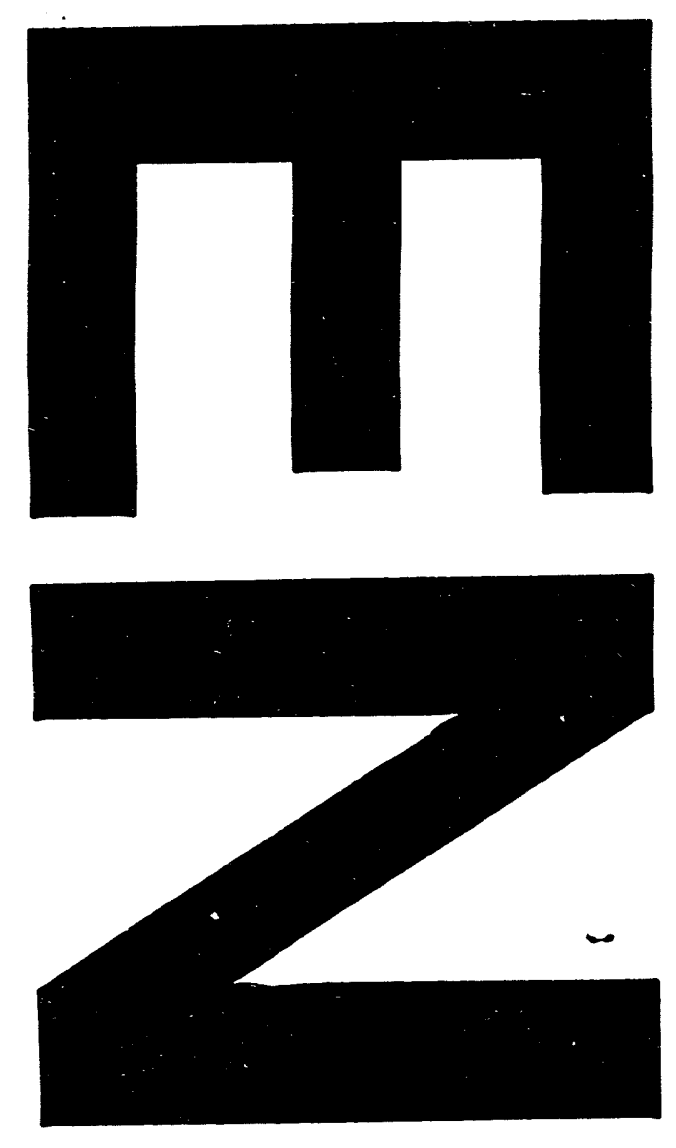

l
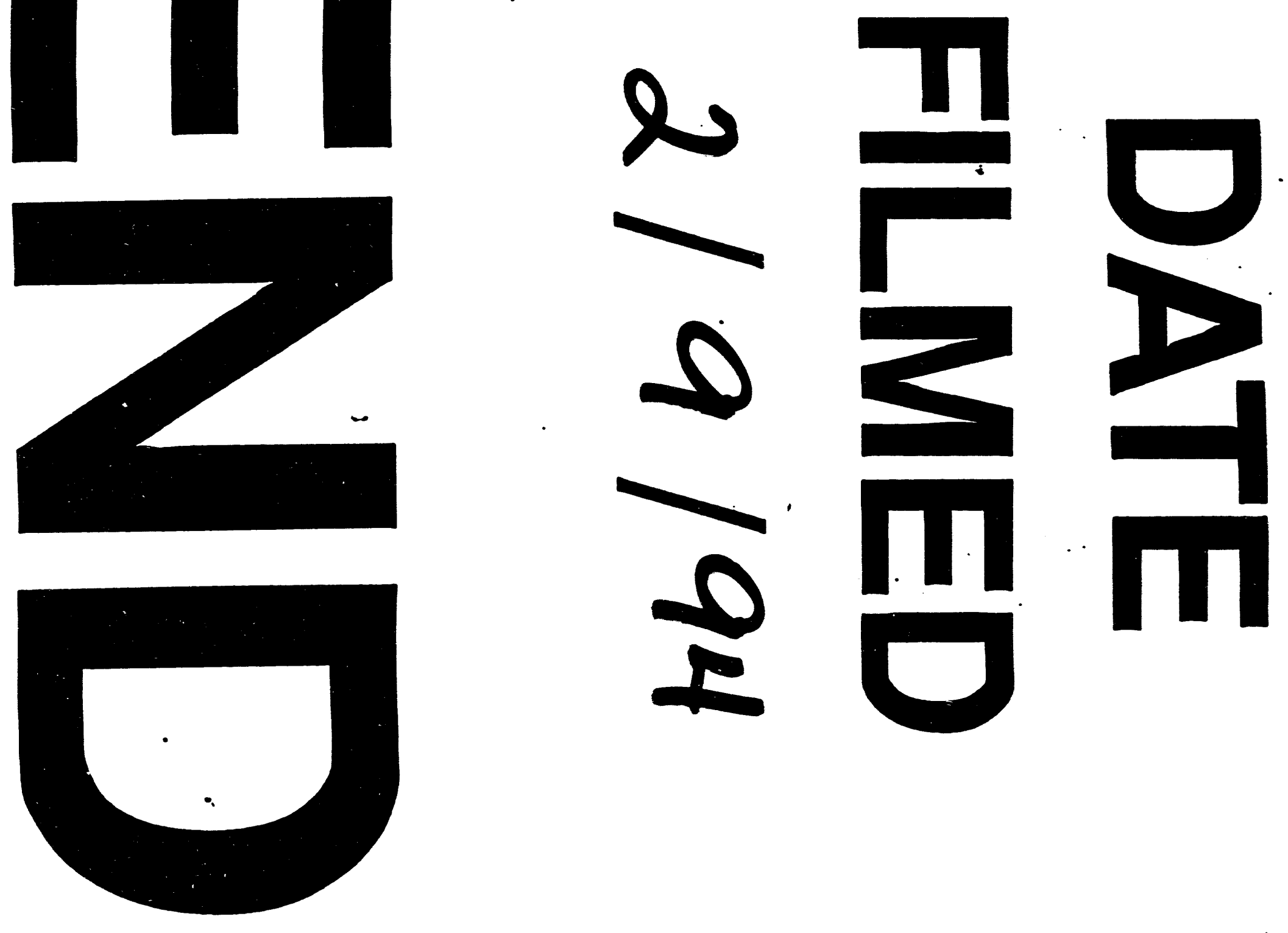
